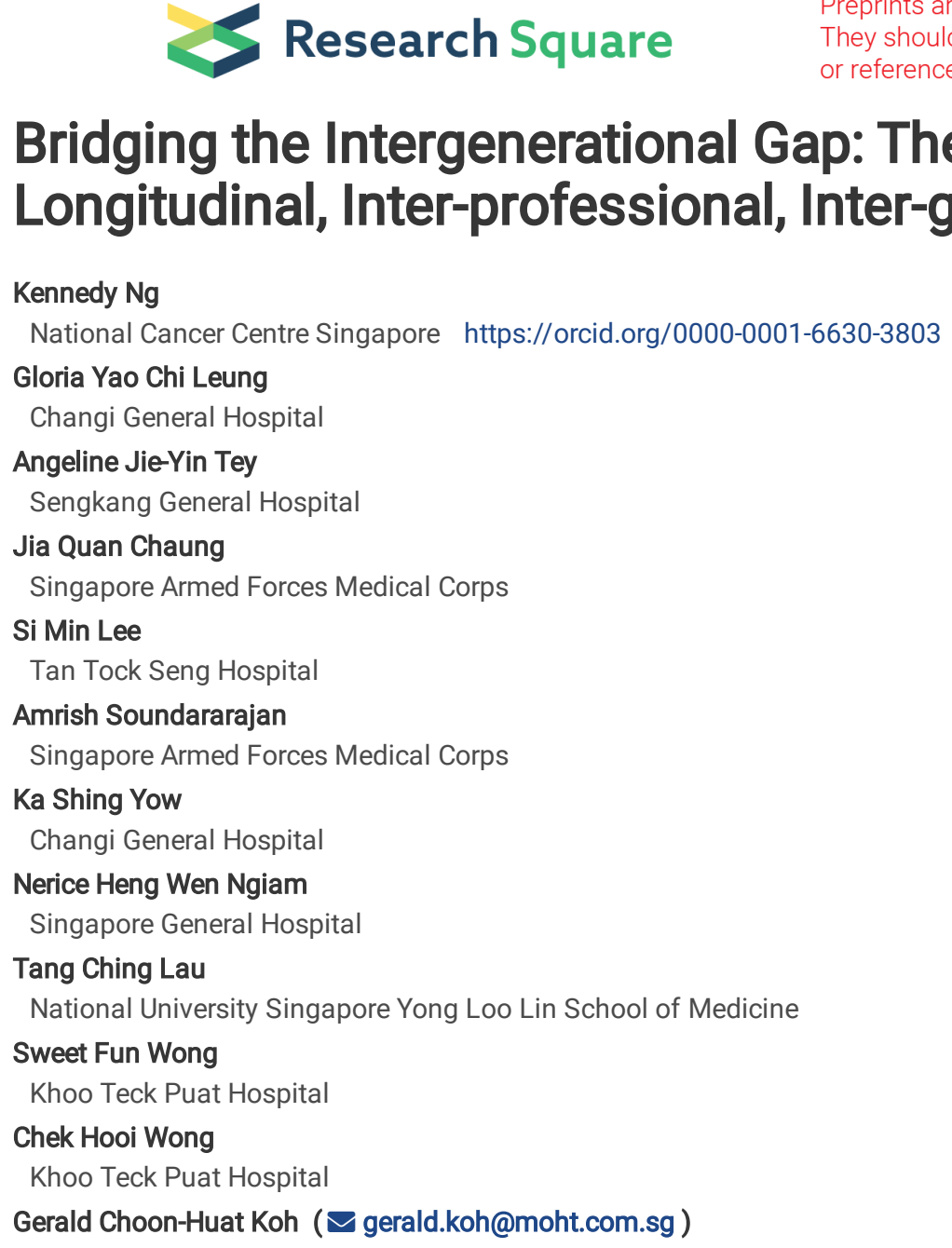

\title{
Bridging the Intergenerational Gap: The Outcomes of a Student-Initiated, Longitudinal, Inter-professional, Inter-generational Home Visit Program
}

Research article

Keywords: Intergeneration interactions, interprofessional, community medicine, ageism

Posted Date: January 20th, 2020

DOI: https://doi.org/10.21203/rs.2.21251/v1

License: (c) (i) This work is licensed under a Creative Commons Attribution 4.0 International License. Read Full License

Version of Record: A version of this preprint was published at BMC Medical Education on May 11th, 2020. See the published version at https://doi.org/10.1186/s12909-020-02064-x. 


\section{Abstract}

Background

The older persons consume disproportionately more healthcare resources than younger persons. Tri-Generational HomeCare (TriGen), a servicelearning program, aimed to reduce hospital admission rates amongst older patients with frequent admissions. The authors evaluated the educational and patient outcomes of TriGen.

Methods

Teams consisting of healthcare undergraduates and lay volunteers - secondary school (SS) students - performed fortnightly home visits to patients over 6 months. Self-administered scales were used to evaluate the educational outcomes. Patients' satisfaction and clinical outcomes were also assessed.

Results

226 healthcare undergraduates and 359 SS students participated in the program from 2015 to 2018 . Response rates were $80.1 \%$ and $62.4 \%$ respectively. 106 patients participated in TriGen. There was a statistically significant increase in Kogan's Attitudes towards Old Persons scores preand post-intervention for healthcare undergraduates and SS students with a mean increase of $12.8(95 \% \mathrm{Cl}: 9.5-16.2, \mathrm{p}<0.001)$ and $8.3(95 \% \mathrm{Cl}$ : 6.2 $-10.3, p<0.001)$ respectively. There was a statistically significant increase in Palmore FAQ score pre- and post-intervention for SS students. Most volunteers reported that TriGen was beneficial across all nine domains assessed. There was a statistically significant decrease in hospital admission rates $(p=0.006)$ and emergency department visits $(p=0.004)$ during the 6 -month period before and after the program. 51 patients answered the patient feedback survey. Of this, more than $80 \%$ reported feeling less lonely and happier.

Conclusion

TriGen, a student-initiated, longitudinal, inter-generational service-learning program consisting of lay students and healthcare undergraduates can reduce ageism, develop soft skills, inculcate values amongst lay volunteers (SS students) and healthcare undergraduates. In addition, TriGen potentially reduces hospital admissions and emergency department visits, and loneliness amongst frequently admitted older patients.

\section{Background}

The demographic landscape in Singapore is aging. By 2030, this is estimated to nearly double to 900,000 , or $25 \%$ of the resident population (1). This poses many challenges. Even though a relatively small proportion of the population, older persons account for a disproportionate $32.0 \%$ of admissions to acute hospitals and $81.0 \%$ of admissions to community hospitals (2). Acute hospitalizations are also associated with complications including nosocomial infections and prolonged hospital stays amongst older adults (3).

Hence, there is increasing interest in avoiding acute hospitalization and promoting community-based healthcare to meet healthcare needs of complex patients (4-6). The advantages of community-based healthcare include extending the reach of healthcare professionals to those who might otherwise not have sought necessary healthcare (3), by forming a stronger therapeutic relationship between the healthcare professional and the individual (7), and by allowing healthcare professionals to better able to tackle individuals' problems holistically and not just medically (8, 9 ). If healthcare professionals can identify and address unmet needs in the community, they can potentially prevent avoidable hospital admissions.

Another approach is to reduce loneliness amongst the older persons. It has been shown that loneliness was associated with hospital admissions, increased lengths of stay and overutilization of healthcare resources, (10) as well as increased mortality and morbidity. (11) We propose that the therapeutic relationship between healthcare professionals, lay volunteers and the older patients can reduce loneliness.

Another proposed intervention is to reduce ageism amongst healthcare undergraduates and the community. It has been shown that ageism amongst healthcare professionals leads to lower quality of care for the older persons $(12,13)$, possibly due to undertreatment by healthcare professionals (14). The presence of ageist attitudes amongst healthcare professionals may lead to the older persons feeling powerless and having decreased selfesteem and discourage them from candidly sharing their concerns, preferences and needs (15). Moreover, per Jackson, Sarah E et al., perceived age discrimination was associated with increased risk of serious health problems among older persons living in England (16). Fortunately, studies have also found that ageism amongst healthcare professionals and non-healthcare professionals can be overcome with education on the aging process (17-19) and positive experiences in interacting with the older persons (19-21). Reducing ageism with these strategies may lead to an increased interest in caring for the older persons (18) and could potentially improve overall quality of care. We opted to target healthcare undergraduates as it is crucial to change the attitudes of healthcare professionals early in their education, since there are studies suggesting that ageism can be amplified as healthcare professionals gain more work experience (22).

Developing empathy, social awareness and other intangible soft skills and values (e.g. teamwork, communication skills, responsibility) amongst the healthcare undergraduates and SS students is also important. It has been shown that empathy level decreases with increasing years of training and education amongst healthcare students and professionals (23-26). This leads to poorer care and patient outcomes. With greater empathy and

Page 2/27 
social awareness, healthcare undergraduates and SS students will be equipped to provide patient-centered care, which has been shown to reduce healthcare utilization and improve health outcomes $(27,28)$.

Tri-Generational HomeCare (TriGen) was conceived to reduce unnecessary acute healthcare resources utilization amongst the older persons. It leverages on the aforementioned interventions: 1) community-based care, 2) reducing loneliness amongst the older person, 3) reducing ageism, 4) development of empathy, social awareness and other intangible soft skills and values amongst secondary school students from the community and healthcare undergraduates. TriGen is based on the service-learning model, with the dual objectives of service to the community and learning by the participants $(29,30)$, and empowers volunteers (healthcare undergraduates and SS students) to provide holistic care and companionship to at-risk older adults. It also provides a structured learning experience where participants can reflect on and learn from their experiences with the aim of reducing ageism, development of soft skills like empathy, social awareness and inculcating values.

\section{Program Description}

TriGen is a collaboration between the National University of Singapore Yong Loo Lin School of Medicine (NUS YLLSoM), Khoo Teck Puat Hospital (KTPH), a regional hospital situated in the Northern part of Singapore, and North West Community Development Council (NWCDC), a social organization based in the northwestern district of Singapore. KTPH developed the aging-in-place (AIP) program that aims to reduce readmission rates amongst high consumers of healthcare, defined as those admitted into hospital three or more times over a six-month period.

TriGen, as the name suggests, involves participants across three generations: i) older patients who are enrolled in the AIP program; ii) healthcare undergraduate enrolled in Medicine, Nursing, Pharmacy, Social Work, Physiotherapy or Occupational Therapy course from various higher educational institutions in Singapore; and iii) SS student, who come mostly from schools in the North-western district.

The learning objectives for healthcare undergraduates and SS students can be summarized in Table 1. 
Table 1

Learning objectives for TriGen participants and aspects of programmes to meet these objectives

\begin{tabular}{|c|c|c|}
\hline \multirow{2}{*}{$\begin{array}{l}\text { Aims for } \\
\text { participants }\end{array}$} & \multicolumn{2}{|l|}{ Aspects of TriGen fulfilling the aims } \\
\hline & Healthcare undergraduates & Secondary school students \\
\hline $\begin{array}{l}\text { Interprofessional } \\
\text { learning }\end{array}$ & $\begin{array}{l}\text { Pre-visitation training } \\
\text { Healthcare undergraduates completed the pre-visitation training in } \\
\text { interprofessional groups } \\
\text { During visitations } \\
\text { Healthcare undergraduates worked in interprofessional groups of } \\
\text { two to three undergraduates to conduct home visits } \\
\text { During conference sessions } \\
\text { Multi-disciplinary meetings with healthcare professionals from } \\
\text { KTPH (including doctors, nurses, pharmacists and social workers) } \\
\text { where students present their assessment of the older persons they } \\
\text { have visited and their proposed management plan, with the KTPH } \\
\text { faculty will provide additional input and guidance }\end{array}$ & \\
\hline $\begin{array}{l}\text { Increasing } \\
\text { knowledge of the } \\
\text { aging process, } \\
\text { reducing ageist } \\
\text { attitudes, basic } \\
\text { caregiving skills }\end{array}$ & $\begin{array}{l}\text { Pre-visitation training } \\
\text { Lectures on common medical conditions in the older person and } \\
\text { the normal aging process } \\
\text { Lectures and workshop addressing possible difficulties in } \\
\text { communicating with older persons and role play on strategies to } \\
\text { overcome them } \\
\text { Role-playing activities to help students understand the sensory } \\
\text { deficits and other problems commonly faced by the older persons, } \\
\text { and strategies to overcome these problems } \\
\text { Occupational Therapist / Physiotherapist-led hands-on session } \\
\text { exploring mobility aids and skills on transfer of the older persons } \\
\text { Workshop teaching basic caregiving skills e.g. measurement of } \\
\text { blood pressure and capillary blood glucose (for non-medical } \\
\text { healthcare undergraduates) } \\
\text { During visitations } \\
\text { Interacting with older persons during home visits, including } \\
\text { monitoring of patients' vital parameters, patient education, } \\
\text { medication reconciliation, befriending and social activities, cleaning } \\
\text { up of the house, coordination of medical/social services }\end{array}$ & $\begin{array}{l}\text { Pre-visitation training } \\
\text { Lecture on aging population trends in Singapore } \\
\text { Workshop addressing possible difficulties in } \\
\text { communicating with older persons and role play } \\
\text { on strategies to overcome them } \\
\text { Role-playing activities to help students } \\
\text { understand the sensory deficits and other } \\
\text { problems commonly faced by the older persons, } \\
\text { and strategies to overcome these problems } \\
\text { Workshop teaching basic caregiving skills e.g. } \\
\text { measurement of blood pressure and capillary } \\
\text { blood glucose } \\
\text { During visitations } \\
\text { Interacting with older persons during home } \\
\text { visits, including monitoring of patients' vital } \\
\text { parameters, patient education, befriending and } \\
\text { social activities, cleaning up of the house, under } \\
\text { the leadership of the healthcare undergraduates } \\
\text { Reflections and debrief sessions held after each } \\
\text { home visit, facilitated by the healthcare } \\
\text { undergraduates, guided by a series of lesson plans } \\
\text { ranging from dementia to communication with the } \\
\text { older persons }\end{array}$ \\
\hline $\begin{array}{l}\text { Other knowledge } \\
\text { and skills }\end{array}$ & $\begin{array}{l}\text { Pre-visitation training } \\
\text { Lecture on community resources available to less privileged } \\
\text { populations and who to refer them for help } \\
\text { Lesson on how to provide counseling on lifestyle and diet } \\
\text { Workshop imparting leadership skills, facilitation skills } \\
\text { During visitations } \\
\text { Leading groups of secondary school students during visitations, } \\
\text { facilitating their interactions with older persons } \\
\text { Facilitating reflections and debrief sessions for the secondary } \\
\text { school students after each home visit, guided by a series of lesson } \\
\text { plans } \\
\text { Collaborating with the healthcare professionals from AIP } \\
\text { During conference sessions } \\
\text { Presenting on the issues faced by the older persons they visited to } \\
\text { healthcare professionals to update on progress and seek } \\
\text { professional input }\end{array}$ & $\begin{array}{l}\text { Pre-visitation training } \\
\text { Lectures on how to provide counseling on } \\
\text { lifestyle and diet } \\
\text { During visitations } \\
\text { Taking vitals under the supervision of the } \\
\text { healthcare undergraduates } \\
\text { Organising of activities to engage the older } \\
\text { persons with, e.g. singing, lantern making, } \\
\text { gardening } \\
\text { Reflections sessions held after each home visit, } \\
\text { facilitated by the healthcare undergraduates }\end{array}$ \\
\hline
\end{tabular}

The program begins with the healthcare undergraduates and SS students undergoing training sessions to prepare them for visitations. Each team comprised two to three undergraduates from different disciplines, and three to five SS students. Each team was allocated one to two patients and fortnightly visits were conducted over six months. Twice over these six months, after the first few visits and again nearing the conclusion of the visitation period, there will be multi-disciplinary meetings where the healthcare undergraduates present on issues faced by the older patients to healthcare professionals from KTPH, including doctors, pharmacists, nurses and social workers, and sought advice on the most appropriate management for these patients.

In this paper, we present the educational and patient outcomes of this student-initiated, home-based, inter-generational, inter-professional, longitudinal service-learning program focused on older patients who have frequent hospital readmissions.

\section{Methods}

\section{Survey Instruments}

Page $4 / 27$ 
For the volunteers, 3 sets of self-administered scales were used: Kogan Attitude towards Old People Scale (KOP) (31) to assess ageist attitudes, Palmore Facts on Aging Quiz (Palmore FAQ) (32) to assess knowledge of older persons, and Fund for the Improvement of Post-Secondary Education Survey Instrument (FIPSE) (33) to assess pedagogical value.

The KOP consists of 17 paired statements on a 5-point Likert scale, one of each pair positively framed and the other negatively framed. Respondents were asked to indicate the level to which they agree or disagree using a 5-point Likert scale. The scores range from 34 to 170 with a neutral score of 102. A higher score indicates a less negative attitude towards older persons.

The Palmore FAQ consists of 25 true/false statements that query health and socioeconomic information about the elderly in the United States. We adapted it to the local context. The scale is scored with 1 point given for every correct answer, with a maximum score of 25 . A higher score indicates better knowledge about the older persons.

The FIPSE comprises nine domains including leadership, communication skills, teamwork, critical thinking skills, ability to identify social issues, actions skills (i.e. the abilities to take action and take on new responsibilities), the ability to see consequences, the acquisition of knowledge, the application of knowledge. Respondents were asked to answer the questions using a 5-point Likert scale ranging from strongly agree, agree, neutral, disagree, strongly disagree.

For the patients, a patient feedback survey consisting of quantitative questions and open-ended questions was administered to the patients.

All scales were administered at two time points (pre- and post-intervention) except the FIPSE and patient feedback survey which were only administered post-intervention.

Number of hospital admissions and emergency department visits by the patients under TriGen during the 6-months period before and after the program was collected.

\section{Procedure}

We obtained ethical approval from the NUS institutional review board and KTPH domain specific review board to evaluate the learning outcomes and the patient outcome of the TriGen respectively. Study participation for both the volunteers and patients was entirely voluntary and anonymous. We took informed consent from both the healthcare undergraduate and the patients and parental consent for the SS students as they were under 21 years-old at the start of the program. Non-participation in the study did not impact students' evaluations. Patients who did not participate in the study continued to receive care from TriGen. There were no incentives provided to all participants for completing the questionnaires.

\section{Statistical Analysis}

Statistical analysis was carried out on the baseline and post-intervention questions for the aforementioned scales. A paired t-test comparing baseline and post-intervention responses was computed for each survey item to determine significant attitude differences ( $p \leq 0.05$ ). One-way ANNOVA was performed to assess for demographic factors that correlated with pre-intervention and magnitude of change in KOP, FAQ, RIPLS scores. We also computed descriptive statistics for the FIPSE and used chi-square analysis to compare the self-reported learning in the nine domains between genders, between pre-clinical (first- and second-year) and clinical (third- to fifth-year) students. We used logistic regression to adjust for clinical exposure when comparing learning between genders amongst healthcare undergraduates and to adjust for age when comparing learning between genders amongst SS students. The Wilcoxon signed-rank test was used to evaluate the difference between the number of emergency department visits and hospital admissions in the 6 months period before and after the program.

For all statistical analyses, we used Statistical Package for Social Sciences (SPSS, Version 23.0, Chicago, Illinois).

\section{Results}

There is a total of 226 healthcare undergraduates and 359 SS students who participated in the program from 2015 to 2018 . Response rate was $80.1 \%$ and $62.4 \%$ amongst the healthcare undergraduates and SS students respectively. Table 2 describes the profile of the participants. 
Table 2

Demographic profile of students who participated in the TriGen Programme from 2014-2017.

\begin{tabular}{|c|c|c|}
\hline & Healthcare undergraduates & Secondary school students \\
\hline Total number of participants & 226 & 359 \\
\hline Number of respondents & 181 & 224 \\
\hline Response rate & $80.1 \%$ & $62.4 \%$ \\
\hline Median Age (Range) & $21(18-41)$ & $15(13-17)$ \\
\hline \multicolumn{3}{|l|}{ Gender } \\
\hline Male & $68(37.6 \%)$ & $79(35.3 \%)$ \\
\hline Female & $113(62.4 \%)$ & $145(64.7 \%)$ \\
\hline \multicolumn{3}{|l|}{ Faculty/School } \\
\hline & Medicine: 57 (31.5\%) & West Spring Secondary School: 80 (35.7\%) \\
\hline & Nursing: 23 (12.7\%) & Yishun Secondary School: 112 (50\%) \\
\hline & Pharmacy: 76 (42.0\%) & Orchid Park Secondary School: 25 (11.2\%) \\
\hline & Social Work: 20 (11.0\%) & Chung Cheng High School: 1 (0.4\%) \\
\hline & Physiotherapy and Occupational Therapy: 5 (2.8\%) & Anglo-Chinese School (Independent): 3 (1.3\%) \\
\hline & & Eunoia Junior College: 3 (1.3\%) \\
\hline \multicolumn{3}{|l|}{ Year of study } \\
\hline Year 1 & $72(39.8 \%)$ & - \\
\hline Year 2 & $41(22.7 \%)$ & $37(16.5 \%)$ \\
\hline Year 3 & $52(28.7 \%)$ & $178(79.5 \%)$ \\
\hline Year 4 & $14(7.7 \%)$ & $3(1.3 \%)$ \\
\hline Year 5 & $2(1.7 \%)$ & $6(2.7 \%)$ \\
\hline \multicolumn{3}{|l|}{ Living with grandparents } \\
\hline Yes & $25(13.8 \%)$ & 49 (21.9\%) \\
\hline No & $156(86.2 \%)$ & $175(78.1 \%)$ \\
\hline \multicolumn{3}{|c|}{ Involved in volunteer work with the older person } \\
\hline Yes & $131(72.4 \%)$ & $123(54.9 \%)$ \\
\hline No & $50(27.6 \%)$ & $101(45.1 \%)$ \\
\hline \multicolumn{3}{|l|}{ Hours spent on CIP } \\
\hline Excluding training & & $410 \min$ \\
\hline Inclusive of training & & $480 \mathrm{~min}$ \\
\hline \multicolumn{3}{|l|}{ Previous IPE activities } \\
\hline \multicolumn{3}{|c|}{ Yes: $117(64.6 \%)$} \\
\hline & No: 64 (35.4\%) & \\
\hline
\end{tabular}

\section{Kogan's Old People score}

\section{Healthcare undergraduates}

There was a statistically significant increase in KOP score pre- and post-intervention for healthcare undergraduates with a mean increase of 12.8 (95\% Cl: 9.46-16.2, p < 0.001). (Table 3) This increase was found in all groups of undergraduates. All subgroups analysed had a statistically significant increase in KOP score pre- and post-intervention. There was a statistically significant difference in pre-intervention KOP score between 
those who stay with their grandparents and those who do not as determined by one-way ANOVA $(F(1,206)=4.778, p=0.03)$. A Tukey post hoc test revealed that undergraduates who stay with their grandparents have higher pre-intervention KOP scores (i.e. have less ageist attitudes) (mean score 138.3, 95\% Cl: 132.5-144.2) as compared to those who do not (mean score 132.1, 95\% Cl: 128.9-135.3). There was no statistically significant difference for pre-intervention KOP found for different genders (male versus female), seniority (preclinical versus clinical), previous or current volunteer work involving the older persons.

Table 3

KOP score in university healthcare undergraduates.

\begin{tabular}{|c|c|c|c|c|c|c|c|}
\hline Group & No. & $\begin{array}{l}\text { Pre-Intervention } \\
\text { Score (mean, } \\
95 \% \mathrm{Cl})\end{array}$ & $\begin{array}{l}\text { P-value (comparison between } \\
\text { groups for pre-intervention } \\
\text { score) }\end{array}$ & $\begin{array}{l}\text { Post-Intervention } \\
\text { Score (mean, } \\
95 \% \mathrm{Cl} \text { ) }\end{array}$ & $\begin{array}{l}\text { Mean } \\
\text { Difference }\end{array}$ & $\begin{array}{l}\mathrm{P} \text { - } \\
\text { value }\end{array}$ & $\begin{array}{l}\text { Comparison } \\
\text { between } \\
\text { groups }\end{array}$ \\
\hline All University Students & 172 & $\begin{array}{l}133.0(130.1- \\
135.9)\end{array}$ & & $\begin{array}{l}145.7(143.3- \\
148.1)\end{array}$ & $\begin{array}{l}12.8(9.4- \\
16.2)\end{array}$ & $\begin{array}{l}<.0001 \\
0\end{array}$ & \\
\hline Gender & & & 0.26 & & & & 0.075 \\
\hline Male & 64 & $\begin{array}{l}135.5(131.2- \\
139.8)\end{array}$ & & $\begin{array}{l}144.3(139.6- \\
148.9)\end{array}$ & $\begin{array}{l}8.8(3.6- \\
14.0)\end{array}$ & 0.001 & \\
\hline Female & 108 & $\begin{array}{l}131.5(127.6- \\
135.3)\end{array}$ & & $\begin{array}{l}146.6(143.9- \\
149.3)\end{array}$ & $\begin{array}{l}15.1 \\
(10.7- \\
19.6)\end{array}$ & $\begin{array}{l}< \\
0.0001\end{array}$ & \\
\hline Year of Study & & & 0.15 & & & & 0.43 \\
\hline Year 1 and 2 & 108 & $\begin{array}{l}134.8(130.6- \\
138.9)\end{array}$ & & $\begin{array}{l}146.5(143.3- \\
149.7)\end{array}$ & $\begin{array}{l}11.7(7.1- \\
16.4)\end{array}$ & $\begin{array}{l}<.0001 \\
0\end{array}$ & \\
\hline Year 3 to 5 & 64 & $\begin{array}{l}130.0(126.7- \\
133.2)\end{array}$ & & $\begin{array}{l}144.5(140.9- \\
148.1)\end{array}$ & $\begin{array}{l}14.5(9.7- \\
19.3)\end{array}$ & $\dot{0} 0001$ & \\
\hline Faculty & & & 0.78 & & & & 0.53 \\
\hline Medical & 57 & $\begin{array}{l}132.8(126.1- \\
139.5)\end{array}$ & & $\begin{array}{l}148.9(144.9- \\
153.0)\end{array}$ & $\begin{array}{l}16.1(8.4- \\
23.9)\end{array}$ & $<.0001$ & \\
\hline Nursing & 23 & $\begin{array}{l}134.2(128.8- \\
139.7)\end{array}$ & & $\begin{array}{l}147.7(142.9- \\
152.4)\end{array}$ & $\begin{array}{l}13.4(8.3- \\
18.6)\end{array}$ & $\begin{array}{l}<.0001 \\
0\end{array}$ & \\
\hline Pharmacy & 68 & $\begin{array}{l}132.5(128.5- \\
136.6)\end{array}$ & & $\begin{array}{l}141.7(137.3- \\
146.1)\end{array}$ & $\begin{array}{l}9.18 \\
(4.00- \\
14.36)\end{array}$ & 0.001 & \\
\hline Social Work & 19 & $\begin{array}{l}131.8(125.5- \\
138.1)\end{array}$ & & $\begin{array}{l}146.6(141.1- \\
152.1)\end{array}$ & $\begin{array}{l}14.79 \\
(7.63- \\
21.95)\end{array}$ & $\begin{array}{l}<.0001 \\
0\end{array}$ & \\
\hline Therapist & 5 & $\begin{array}{l}139.4(133.0- \\
145.8)\end{array}$ & & $\begin{array}{l}151.6(142.7- \\
160.5)\end{array}$ & $\begin{array}{l}12.2(3.0- \\
21.4)\end{array}$ & 0.021 & \\
\hline $\begin{array}{l}\text { Living with } \\
\text { grandparents }\end{array}$ & & & 0.032 & & & & 0.65 \\
\hline Yes & 24 & $\begin{array}{l}138.3(132.5- \\
144.2)\end{array}$ & & $\begin{array}{l}149.1(143.2- \\
155.1)\end{array}$ & $\begin{array}{l}10.8 \\
(2.73- \\
18.9)\end{array}$ & 0.011 & \\
\hline No & 148 & $\begin{array}{l}132.1(128.9 \\
-135.3)\end{array}$ & & $\begin{array}{l}145.2(142.5- \\
147.8)\end{array}$ & $\begin{array}{l}13.1(9.3- \\
16.8)\end{array}$ & $\begin{array}{l}<.0001 \\
0.00\end{array}$ & \\
\hline $\begin{array}{l}\text { Have you volunteered } \\
\text { in an old person } \\
\text { facility? }\end{array}$ & & & 0.97 & & & & 0.13 \\
\hline Yes & 127 & $\begin{array}{l}132.9(129.2- \\
136.5)\end{array}$ & & $\begin{array}{l}147.2(144.2- \\
150.1)\end{array}$ & $\begin{array}{l}14.3(9.9- \\
18.7)\end{array}$ & $\begin{array}{l}<.0001 \\
0.00\end{array}$ & \\
\hline No & 45 & $\begin{array}{l}133.2(129.0- \\
137.4)\end{array}$ & & $\begin{array}{l}141.6(137.7- \\
145.6)\end{array}$ & $\begin{array}{l}8.4(4.5- \\
12.3)\end{array}$ & $\begin{array}{l}< \\
0.0001\end{array}$ & \\
\hline
\end{tabular}

Pre-intervention Kogan's scores is weakly positively associated with post-intervention Kogan's scores $(r=0.177, p=0.020)$ and moderately negatively associated with the difference in Kogan's scores $(r=-0.724, p<0.001)$. 
There was a statistically significant increase in KOP score pre- and post-intervention for SS students with a mean increase of 8.3 (95\% CI: $6.2-10.3$, $p<0.001$ ). (Table 4) All subgroups analysed had a statistically significant increase in KOP score pre- and post-intervention. There was no statistically significant difference for pre-intervention KOP found between the different genders (male versus female), secondary schools, seniority (lower secondary versus upper secondary), whether they are living with their grandparents or having previous volunteering experience in an old person facility. 
KOP score in secondary school students.

\begin{tabular}{|c|c|c|c|c|c|c|c|}
\hline Group & No. & $\begin{array}{l}\text { Pre-Intervention } \\
\text { Score (mean, } \\
95 \% \mathrm{Cl} \text { ) }\end{array}$ & $\begin{array}{l}\text { P-value (comparison between } \\
\text { groups for pre-intervention } \\
\text { score) }\end{array}$ & $\begin{array}{l}\text { Post-Intervention } \\
\text { Score (mean, } \\
95 \% \mathrm{Cl} \text { ) }\end{array}$ & $\begin{array}{l}\text { Mean } \\
\text { Difference }\end{array}$ & $\begin{array}{l}\mathrm{P} \text { - } \\
\text { value }\end{array}$ & $\begin{array}{l}\text { Comparison } \\
\text { between } \\
\text { groups }\end{array}$ \\
\hline All secondary school & 224 & $\begin{array}{l}127.4(125.6- \\
129.2)\end{array}$ & & $\begin{array}{l}135.7(134.1- \\
137.3)\end{array}$ & $\begin{array}{l}8.3(6.2- \\
10.3)\end{array}$ & $<.001$ & \\
\hline Gender & & & 0.27 & & & & 0.55 \\
\hline Male & 79 & $\begin{array}{l}128.78(125.21- \\
132.36)\end{array}$ & & $\begin{array}{l}136.19(133.68- \\
138.71)\end{array}$ & $\begin{array}{l}7.41 \\
(3.46- \\
11.35)\end{array}$ & $\begin{array}{l}<.001 \\
0\end{array}$ & \\
\hline Female & 145 & $\begin{array}{l}126.67(14.66- \\
128.67)\end{array}$ & & $\begin{array}{l}135.42(133.07- \\
137.77)\end{array}$ & $\begin{array}{l}8.75 \\
(6.43- \\
11.07)\end{array}$ & $<.001$ & \\
\hline Age & & & 0.12 & & & & 0.69 \\
\hline Younger (Age 13-14) & 63 & $\begin{array}{l}125.16(122.69- \\
127.63)\end{array}$ & & $\begin{array}{l}134.05(130.47- \\
137.63)\end{array}$ & $\begin{array}{l}8.89 \\
(5.18- \\
12.59)\end{array}$ & $<.001$ & \\
\hline Older (Age 15-17) & 161 & $\begin{array}{l}128.30(125.98- \\
130.61)\end{array}$ & & $\begin{array}{l}136.34(134.33- \\
138.34)\end{array}$ & $\begin{array}{l}8.04 \\
(5.59- \\
10.48)\end{array}$ & $<.001$ & \\
\hline School & & & 0.43 & & & & 0.54 \\
\hline School 1 (Westspring) & 80 & $\begin{array}{l}127.48(124.31- \\
130.64)\end{array}$ & & $\begin{array}{l}137.84(135.24- \\
140.44)\end{array}$ & $\begin{array}{l}10.36 \\
(6.83- \\
13.90)\end{array}$ & $\hat{0} .001$ & \\
\hline School 2 (Yishun Sec) & 112 & $\begin{array}{l}126.30(123.83- \\
128.76)\end{array}$ & & $\begin{array}{l}133.82(131.11- \\
136.53)\end{array}$ & $\begin{array}{l}7.53 \\
(4.49- \\
10.57)\end{array}$ & $<.001$ & \\
\hline Others & 32 & $\begin{array}{l}131.10(126.47- \\
135.91)\end{array}$ & & $\begin{array}{l}136.88(132.71- \\
141.04)\end{array}$ & $\begin{array}{l}5.69 \\
(2.01- \\
9.36)\end{array}$ & 0.004 & \\
\hline $\begin{array}{l}\text { Living with } \\
\text { grandparents }\end{array}$ & & & 0.45 & & & & 0.84 \\
\hline Yes & 49 & $\begin{array}{l}126.12(122.70- \\
129.54)\end{array}$ & & $\begin{array}{l}133.96(131.02- \\
136.90)\end{array}$ & $\begin{array}{l}7.84 \\
(4.36- \\
11.32)\end{array}$ & $<.001$ & \\
\hline No & 175 & $\begin{array}{l}127.78(125.69- \\
129.86)\end{array}$ & & $\begin{array}{l}136.18(134.08- \\
138.27)\end{array}$ & $\begin{array}{l}8.40 \\
(5.98- \\
10.82)\end{array}$ & $\dot{0} .001$ & \\
\hline $\begin{array}{l}\text { Have you volunteered } \\
\text { in an old person } \\
\text { facility? }\end{array}$ & & & 0.37 & & & & 0.26 \\
\hline Yes & 123 & $\begin{array}{l}128.15(125.94- \\
130.37)\end{array}$ & & $\begin{array}{l}135.41(132.79- \\
138.02)\end{array}$ & $\begin{array}{l}7.25 \\
(4.56- \\
9.94)\end{array}$ & $<.001$ & \\
\hline No & 101 & $\begin{array}{l}126.52(123.59- \\
129.44)\end{array}$ & & $\begin{array}{l}136.04(133.78- \\
138.30)\end{array}$ & $\begin{array}{l}9.52 \\
(6.40- \\
12.65)\end{array}$ & $<.001$ & \\
\hline $\begin{array}{l}\text { Do you have any } \\
\text { siblings? }\end{array}$ & & & 0.56 & & & & 0.36 \\
\hline Yes & 185 & $\begin{array}{l}127.17(125.18- \\
129.17)\end{array}$ & & $\begin{array}{l}135.89(133.98- \\
137.81)\end{array}$ & $\begin{array}{l}8.72 \\
(6.41- \\
11.03)\end{array}$ & $<.001$ & \\
\hline No & 39 & $\begin{array}{l}128.56(124.52- \\
132.61)\end{array}$ & & $\begin{array}{l}134.74(130.31- \\
139.18)\end{array}$ & $\begin{array}{l}6.18 \\
(2.11- \\
10.25)\end{array}$ & 0.004 & \\
\hline
\end{tabular}

Number of hours spent on home visits is weakly positively associated with difference in KOP scores $(r=0.234, p<0.001)$. Pre-intervention Kogan's scores is weakly positively associated with post-intervention Kogan's scores $(r=0.333, p<0.001)$ and moderately negatively associated with the 
difference in Kogan's scores $(r=-0.598, p<0.001)$.

The baseline KOP score of the healthcare undergraduates is significantly higher than that of the secondary school students by $(\mathrm{mean}=6.8,95 \% \mathrm{Cl}$ : 3.7 to $9.9, p<0.001)$, and the increase in KOP score for healthcare undergraduates was also significantly more than that of the secondary school students (mean $=4.6,95 \% \mathrm{Cl}: 0.7$ to $8.4, \mathrm{p}=0.022$ ).

\section{Palmore's Facts of Aging Quiz}

\section{Healthcare undergraduates}

The average pre- and post-intervention Palmore's FAQ score is 15.8 and 16.0 respectively, but there was no significant difference in Palmore's FAQ score $(p=0.112)$. (Table 5) 
Table 5

Palmore score in healthcare undergraduate

\begin{tabular}{|c|c|c|c|c|c|c|c|}
\hline Group & No. & $\begin{array}{l}\text { Pre-Intervention } \\
\text { Score (mean, } \\
95 \% \mathrm{Cl} \text { ) }\end{array}$ & $\begin{array}{l}\text { P-value (comparison between } \\
\text { groups for pre-intervention } \\
\text { score) }\end{array}$ & $\begin{array}{l}\text { Post-Intervention } \\
\text { Score (mean, } \\
95 \% \mathrm{Cl})\end{array}$ & $\begin{array}{l}\text { Mean } \\
\text { Difference }\end{array}$ & $\begin{array}{l}\mathrm{P}- \\
\text { value }\end{array}$ & $\begin{array}{l}\text { Comparison } \\
\text { between } \\
\text { groups }\end{array}$ \\
\hline All university students & 127 & $15.7(15.1-16.2)$ & & $16.2(15.7-16.6)$ & $\begin{array}{l}0.49(- \\
0.095- \\
1.07)\end{array}$ & 0.10 & \\
\hline Gender & & & 0.050 & & & & 0.43 \\
\hline Male & 48 & $16.3(15.5-17.1)$ & & $16.5(15.9-17.1)$ & $\begin{array}{l}0.19(- \\
0.61- \\
0.98)\end{array}$ & 0.64 & \\
\hline Female & 79 & $15.3(14.5-16.1)$ & & $15.9(15.4-16.5)$ & $\begin{array}{l}0.67(- \\
0.14- \\
1.49)\end{array}$ & 0.11 & \\
\hline Year of Study & & & 0.13 & & & & 0.087 \\
\hline Year 1 and 2 & 93 & $16.0(15.4-16.7)$ & & $16.2(15.8-16.7)$ & $\begin{array}{l}0.18(- \\
0.48- \\
0.85)\end{array}$ & 0.59 & \\
\hline Year 3 to 5 & 34 & $14.7(13.5-15.8)$ & & $16.0(15.1-16.8)$ & $\begin{array}{l}1.32 \\
(0.11- \\
2.54)\end{array}$ & 0.034 & \\
\hline Faculty & & & 0.20 & & & & 0.14 \\
\hline Medical & 44 & $16.0(14.9-17.1)$ & & $16.8(16.2-17.4)$ & $\begin{array}{l}0.77 \\
(-0.33- \\
1.87)\end{array}$ & 0.16 & \\
\hline Nursing & 14 & $13.8(11.2-16.3)$ & & $15.5(14.4-16.6)$ & $\begin{array}{l}1.71(- \\
1.05- \\
4.48)\end{array}$ & 0.20 & \\
\hline Pharmacy & 51 & $16.1(15.3-16.8)$ & & $15.7(15.0-16.4)$ & $\begin{array}{l}-0.37(- \\
1.11- \\
0.36)\end{array}$ & 0.31 & \\
\hline Social Work & 13 & $15.2(13.7-16.7)$ & & $16.7(15.4-18.0)$ & $\begin{array}{l}1.54(- \\
0.16- \\
3.24)\end{array}$ & 0.072 & \\
\hline Therapist & 5 & $15.0(13.6-16.4)$ & & $15.6(13.9-17.3)$ & $\begin{array}{l}0.60(- \\
1.98- \\
3.18)\end{array}$ & 0.553 & \\
\hline $\begin{array}{l}\text { Living with } \\
\text { grandparents }\end{array}$ & & & 0.14 & & & & 0.55 \\
\hline Yes & 18 & $16.3(15.3-17.2)$ & & $16.3(15.2-17.5)$ & $\begin{array}{l}0.056(- \\
1.12- \\
1.23)\end{array}$ & 0.922 & \\
\hline No & 109 & $15.6(14.9-16.2)$ & & $16.1(15.7-16.6)$ & $\begin{array}{l}0.56(- \\
0.097- \\
1.22)\end{array}$ & 0.094 & \\
\hline $\begin{array}{l}\text { Have you volunteered } \\
\text { in an old person } \\
\text { facility? }\end{array}$ & & & 0.59 & & & & 0.26 \\
\hline Yes & 95 & $15.8(15.1-16.5)$ & & $16.1(15.7-16.6)$ & $\begin{array}{l}0.30(- \\
0.43- \\
1.02)\end{array}$ & 0.42 & \\
\hline No & 32 & $15.1(14.1-16.2)$ & & $16.2(15.3-17.1)$ & $\begin{array}{l}1.06 \\
(0.17- \\
1.96)\end{array}$ & 0.021 & \\
\hline
\end{tabular}

There is a weak positive correlation between: i) baseline Kogan's and baseline Palmore's scores $(\rho=0.183, p=0.008)$; ii) post-intervention Kogan's and post-intervention Palmore's scores $(\rho=0.373, p<0.001)$; and iii) change in Kogan's and change in Palmore's scores $(\rho=0.266, p<0.001)$. 


\section{Secondary School Students}

There was a statistically significant increase in Palmore FAQ score pre- and post-intervention for SS students with a mean increase of 0.8 ( $95 \%$ Cl: $0.3-1.4, p=0.005$ ). (Table 6) Female students, older students, those who do not live with their grandparents, those who had never volunteered in an old person facility and those who have siblings were associated with a statistically significant increase in Palmore FAQ score. There was no statistically significant difference in pre-intervention Palmore FAQ score between the different genders (male versus female), secondary schools, seniority (lower secondary versus upper secondary), whether they are living with their grandparents or having previous volunteering experience in an old person facility. 
Palmore score in secondary school students

\begin{tabular}{|c|c|c|c|c|c|c|c|}
\hline Group & No. & $\begin{array}{l}\text { Pre-Intervention } \\
\text { Score (mean, } \\
95 \% \mathrm{Cl})\end{array}$ & $\begin{array}{l}\text { P-value (comparison between } \\
\text { groups for pre-intervention } \\
\text { score) }\end{array}$ & $\begin{array}{l}\text { Post-Intervention } \\
\text { Score (mean, } \\
95 \% \mathrm{Cl})\end{array}$ & $\begin{array}{l}\text { Mean } \\
\text { Difference }\end{array}$ & $\begin{array}{l}\mathrm{P}- \\
\text { value }\end{array}$ & $\begin{array}{l}\text { Comparison } \\
\text { between groups } \\
\text { for mean } \\
\text { difference }\end{array}$ \\
\hline All secondary school & 162 & $\begin{array}{l}13.3(12.9- \\
13.8)\end{array}$ & & $14.2(13.6-14.7)$ & $\begin{array}{l}0.81 \\
(0.25- \\
1.38)\end{array}$ & 0.005 & \\
\hline Gender & & & 0.061 & & & & 0.57 \\
\hline Male & 64 & $\begin{array}{l}13.89(13.17- \\
14.61)\end{array}$ & & $\begin{array}{l}14.53(13.79- \\
15.28)\end{array}$ & $\begin{array}{l}0.64 \\
(-0.12- \\
1.40)\end{array}$ & 0.097 & \\
\hline Female & 98 & $\begin{array}{l}12.99(12.38- \\
13.60)\end{array}$ & & $\begin{array}{l}13.92(13.13- \\
14.71)\end{array}$ & $\begin{array}{l}0.93 \\
(0.13- \\
1.73)\end{array}$ & 0.023 & \\
\hline Age & & & 0.15 & & & & 0.21 \\
\hline Younger (Age 13-14) & 32 & $\begin{array}{l}14.03(12.89- \\
15.17)\end{array}$ & & $\begin{array}{l}14.16(12.83- \\
15.48)\end{array}$ & $\begin{array}{l}0.13 \\
(-1.18- \\
1.43)\end{array}$ & 0.85 & \\
\hline Older (Age 15-17) & 130 & $\begin{array}{l}13.18(12.67- \\
13.69)\end{array}$ & & $\begin{array}{l}14.16(13.54- \\
14.78)\end{array}$ & $\begin{array}{l}0.98 \\
(0.36- \\
1.61)\end{array}$ & 0.002 & \\
\hline School & & & 0.23 & & & & 0.15 \\
\hline $\begin{array}{l}\text { School } 1 \\
\text { (Westspring) }\end{array}$ & 52 & $\begin{array}{l}13.77(12.92- \\
14.62)\end{array}$ & & $\begin{array}{l}14.08(13.18- \\
14.97)\end{array}$ & $\begin{array}{l}0.31(- \\
0.66- \\
1.28)\end{array}$ & 0.53 & \\
\hline $\begin{array}{l}\text { School } 2 \text { (Yishun } \\
\text { Sec) }\end{array}$ & 81 & $\begin{array}{l}12.90(12.23- \\
13.57)\end{array}$ & & $\begin{array}{l}13.90(13.09- \\
14.71)\end{array}$ & $\begin{array}{l}1.00 \\
(0.23- \\
1.77)\end{array}$ & 0.011 & \\
\hline Others & 29 & $\begin{array}{l}13.82(12.82- \\
14.84)\end{array}$ & & $\begin{array}{l}15.03(13.57- \\
16.50)\end{array}$ & $\begin{array}{l}1.21 \\
(-0.44- \\
2.85)\end{array}$ & 0.14 & \\
\hline $\begin{array}{l}\text { Living with } \\
\text { grandparents }\end{array}$ & & & 0.75 & & & & 0.22 \\
\hline Yes & 37 & $\begin{array}{l}13.49(12.66- \\
14.31)\end{array}$ & & $\begin{array}{l}13.68(12.52- \\
14.84)\end{array}$ & $\begin{array}{l}0.19(- \\
0.93- \\
1.31)\end{array}$ & 0.73 & \\
\hline No & 125 & $\begin{array}{l}13.30(12.75- \\
13.86)\end{array}$ & & $\begin{array}{l}14.30(13.66- \\
14.95)\end{array}$ & $\begin{array}{l}1.00 \\
(0.35- \\
1.65)\end{array}$ & 0.003 & \\
\hline $\begin{array}{l}\text { Have you volunteered } \\
\text { in an old person } \\
\text { facility? }\end{array}$ & & & 0.66 & & & & 0.49 \\
\hline Yes & 88 & $\begin{array}{l}13.25(12.64- \\
13.86)\end{array}$ & & $\begin{array}{l}13.86(13.10- \\
14.63)\end{array}$ & $\begin{array}{l}0.61 \\
(-0.11- \\
1.33)\end{array}$ & 0.093 & \\
\hline No & 74 & $\begin{array}{l}13.56(12.73- \\
14.19)\end{array}$ & & $\begin{array}{l}14.51(13.69- \\
15.34)\end{array}$ & $\begin{array}{l}1.05 \\
(0.15- \\
1.96)\end{array}$ & 0.023 & \\
\hline $\begin{array}{l}\text { Do you have any } \\
\text { siblings? }\end{array}$ & & & 0.084 & & & & 0.58 \\
\hline Yes & 128 & $\begin{array}{l}13.56(13.02- \\
14.09)\end{array}$ & & $\begin{array}{l}14.28(13.66- \\
14.90)\end{array}$ & $\begin{array}{l}0.73 \\
(0.11- \\
1.34)\end{array}$ & 0.021 & \\
\hline No & 34 & $\begin{array}{l}12.56(11.67- \\
13.45)\end{array}$ & & $\begin{array}{l}13.71(12.41- \\
15.00)\end{array}$ & $\begin{array}{l}1.15 \\
(-0.27- \\
2.57)\end{array}$ & 0.11 & \\
\hline
\end{tabular}


There is no correlation between time spent visiting the elderly and change in Palmore's FAQ scores $(\rho=0.136, p=0.084)$. There is a moderate positive correlation between: i) baseline Kogan's and baseline Palmore's scores $(\rho=0.437, p<0.001)$; and ii) post-intervention Kogan's and postintervention Palmore's scores $(\rho=0.472, p<0.001)$. There is a weak positive correlation between the change in Kogan's and the change in Palmore's scores $(\rho=0.349, p<0.001)$.

Healthcare undergraduates had a significantly higher baseline $(2.5,95 \% \mathrm{Cl}: 1.8$ to $3.1, \mathrm{p}<0.001)$ and post-intervention (1.8, $95 \% \mathrm{Cl}$ : 1.1 to $2.5, \mathrm{p}<$ 0.001) Palmore's FAQ score than SS students. However, the healthcare undergraduates did not have a significant difference in change in Palmore's FAQ score compared to the SS students $(-0.3,95 \% \mathrm{Cl}:-1.1$ to $0.5, \mathrm{p}=0.412)$.

\section{Fund for improving postsecondary education survey}

\section{Healthcare undergraduates}

Most healthcare undergraduates felt that TriGen was beneficial across all nine FIPSE domains. (Table 7) $90-100 \%$ of students reported learning in all 9 domains except for ability to make clinical diagnosis (81.6\%) and apply what they have learnt in the training sessions to the home visits (80.1\%). 
Table 7

FIPSE in Healthcare Undergraduates

\begin{tabular}{|c|c|c|c|c|c|c|c|c|c|c|c|}
\hline \multirow{2}{*}{$\begin{array}{l}\text { Domains of } \\
\text { learning-“" feel } \\
\text { that TriGen has } \\
\text { helped me" }\end{array}$} & \multirow{2}{*}{$\begin{array}{l}\text { Total } \\
\text { no. } \\
(\%), n \\
=196\end{array}$} & \multirow{2}{*}{$\begin{array}{l}\text { Male } \\
\text { students, } \\
\text { no. (\%), } \\
n=68\end{array}$} & \multirow{2}{*}{$\begin{array}{l}\text { Female } \\
\text { students, } \\
\text { no.(\%), n } \\
=122\end{array}$} & \multicolumn{3}{|c|}{ Males versus females } & \multirow{2}{*}{$\begin{array}{l}\text { Year } \\
1 \text { and } \\
2, \text { no. } \\
(\%), n \\
=119\end{array}$} & \multirow{2}{*}{$\begin{array}{l}\text { Year } 3 \\
\text { and } \\
\text { above, } \\
\text { no. } \\
(\%), n \\
=71\end{array}$} & \multicolumn{3}{|c|}{ Pre-clinical versus Clinical } \\
\hline & & & & $\begin{array}{l}\text { Pearson } \\
\text { Chi- } \\
\text { Square }\end{array}$ & $\begin{array}{l}\text { Unadjusted } \\
\text { odds ratio } \\
\text { (OR) (95\% } \\
\text { confidence } \\
\text { interval) }\end{array}$ & $\begin{array}{l}\mathrm{P} \\
\text { value }\end{array}$ & & & $\begin{array}{l}\text { Pearson } \\
\text { Chi- } \\
\text { Square }\end{array}$ & $\begin{array}{l}\text { Unadjusted } \\
\text { odds ratio } \\
\text { (OR) ( } 95 \% \\
\text { confidence } \\
\text { interval) }\end{array}$ & $\begin{array}{l}P \\
\text { value }\end{array}$ \\
\hline \multicolumn{12}{|l|}{ Leadership Skills } \\
\hline $\begin{array}{l}\text { Feel responsible } \\
\text { for others in the } \\
\text { Community }\end{array}$ & $\begin{array}{l}191 \\
(97.4)\end{array}$ & $65(95.6)$ & $\begin{array}{l}120 \\
(98.4)\end{array}$ & 1.31 & $\begin{array}{l}0.361 \\
(0.059- \\
2.22)\end{array}$ & 0.25 & $\begin{array}{l}116 \\
(97.5)\end{array}$ & $\begin{array}{l}69 \\
(97.2)\end{array}$ & 0.015 & $\begin{array}{l}1.12 \\
(0.183- \\
6.88)\end{array}$ & 0.90 \\
\hline $\begin{array}{l}\text { Improve my } \\
\text { leadership skills }\end{array}$ & $\begin{array}{l}188 \\
(95.9)\end{array}$ & $63(92.6)$ & $\begin{array}{l}119 \\
(97.5)\end{array}$ & 2.59 & $\begin{array}{l}0.318 \\
(0.074- \\
1.37)\end{array}$ & 0.11 & $\begin{array}{l}115 \\
(96.6)\end{array}$ & $\begin{array}{l}67 \\
(94.4)\end{array}$ & 0.569 & $\begin{array}{l}1.72 \\
(0.416- \\
7.09)\end{array}$ & 0.45 \\
\hline \multicolumn{12}{|c|}{ Communication Skills } \\
\hline $\begin{array}{l}\text { Participate in } \\
\text { community } \\
\text { affairs }\end{array}$ & $\begin{array}{l}186 \\
(94.9)\end{array}$ & $61(89.7)$ & $\begin{array}{l}119 \\
(97.5)\end{array}$ & 5.38 & $\begin{array}{l}0.22 \\
(0.055- \\
0.88)\end{array}$ & $0.020^{+}$ & $\begin{array}{l}112 \\
(94.1)\end{array}$ & $\begin{array}{l}68 \\
(95.8)\end{array}$ & 0.245 & $\begin{array}{l}0.706 \\
(0.177- \\
2.822)\end{array}$ & 0.62 \\
\hline $\begin{array}{l}\text { Develop } \\
\text { communication, } \\
\text { listening and } \\
\text { negotiation } \\
\text { skills }\end{array}$ & $\begin{array}{l}194 \\
(99.0)\end{array}$ & $67(98.5)$ & $\begin{array}{l}121 \\
(99.2)\end{array}$ & 0.178 & $\begin{array}{l}0.554 \\
(0.034- \\
9.00)\end{array}$ & 0.67 & $\begin{array}{l}119 \\
(100)\end{array}$ & $\begin{array}{l}69 \\
(97.2)\end{array}$ & 3.39 & $\begin{array}{l}1.03 \\
(0.989- \\
1.07)\end{array}$ & 0.066 \\
\hline \multicolumn{12}{|l|}{ Teamwork } \\
\hline Think of others & $\begin{array}{l}192 \\
(98.0)\end{array}$ & $65(95.6)$ & $\begin{array}{l}121 \\
(99.2)\end{array}$ & 2.734 & $\begin{array}{l}0.179 \\
(0.018- \\
1.76)\end{array}$ & 0.098 & $\begin{array}{l}117 \\
(98.3)\end{array}$ & $\begin{array}{l}69 \\
(97.2)\end{array}$ & 0.279 & $\begin{array}{l}1.696 \\
(0.234- \\
12.31)\end{array}$ & 0.60 \\
\hline $\begin{array}{l}\text { Appreciate } \\
\text { teamwork and } \\
\text { cooperation } \\
\text { among peers }\end{array}$ & $\begin{array}{l}194 \\
(99.0)\end{array}$ & $67(98.5)$ & $\begin{array}{l}121 \\
(99.2)\end{array}$ & 0.178 & $\begin{array}{l}0.554 \\
(0.034- \\
9.00)\end{array}$ & 0.67 & $\begin{array}{l}118 \\
(99.2)\end{array}$ & $\begin{array}{l}70 \\
(98.6)\end{array}$ & 0.138 & $\begin{array}{l}1.69 \\
(0.104- \\
27.4)\end{array}$ & 0.71 \\
\hline $\begin{array}{l}\text { Be tolerant of } \\
\text { different people }\end{array}$ & $\begin{array}{l}194 \\
(99.0)\end{array}$ & $67(98.5)$ & $\begin{array}{l}121 \\
(99.2)\end{array}$ & 0.178 & $\begin{array}{l}0.554 \\
(0.034- \\
9.00)\end{array}$ & 0.67 & $\begin{array}{l}118 \\
(99.2)\end{array}$ & $\begin{array}{l}70 \\
(98.6)\end{array}$ & 0.138 & $\begin{array}{l}1.69 \\
(0.104- \\
27.4)\end{array}$ & 0.71 \\
\hline $\begin{array}{l}\text { Respect } \\
\text { different } \\
\text { opinions }\end{array}$ & $\begin{array}{l}194 \\
(99.0)\end{array}$ & $67(98.5)$ & $\begin{array}{l}121 \\
(99.2)\end{array}$ & 0.178 & $\begin{array}{l}0.554 \\
(0.034- \\
9.00)\end{array}$ & 0.67 & $\begin{array}{l}119 \\
(100)\end{array}$ & $\begin{array}{l}69 \\
(97.2)\end{array}$ & 3.39 & $\begin{array}{l}1.03 \\
(0.989- \\
1.07)\end{array}$ & 0.066 \\
\hline Compromise & $\begin{array}{l}189 \\
(96.4)\end{array}$ & $64(94.1)$ & $\begin{array}{l}119 \\
(97.5)\end{array}$ & 1.44 & $\begin{array}{l}0.403 \\
(0.088- \\
1.86)\end{array}$ & 0.23 & $\begin{array}{l}115 \\
(96.6)\end{array}$ & $\begin{array}{l}68 \\
(95.8)\end{array}$ & 0.094 & $\begin{array}{l}1.29 \\
(0.276- \\
5.84)\end{array}$ & 0.76 \\
\hline $\begin{array}{l}\text { Comprehend the } \\
\text { moral and } \\
\text { ethical issues in } \\
\text { health care }\end{array}$ & $\begin{array}{l}183 \\
(93.4)\end{array}$ & $63(92.6)$ & $\begin{array}{l}114 \\
(93.4)\end{array}$ & 0.043 & $\begin{array}{l}0.884 \\
(0.277- \\
2.82)\end{array}$ & 0.84 & $\begin{array}{l}107 \\
(89.9)\end{array}$ & $\begin{array}{l}70 \\
(98.6)\end{array}$ & 5.25 & $\begin{array}{l}0.127 \\
(0.016- \\
1.00)\end{array}$ & $0.022^{*}$ \\
\hline \multicolumn{12}{|c|}{ Ability to see consequences } \\
\hline $\begin{array}{l}\text { Think about the } \\
\text { future }\end{array}$ & $\begin{array}{l}187 \\
(95.4)\end{array}$ & $62(91.2)$ & $\begin{array}{l}119 \\
(97.5)\end{array}$ & 3.92 & $\begin{array}{l}0.261 \\
(0.063- \\
1.08)\end{array}$ & 0.048 & $\begin{array}{l}114 \\
(95.8)\end{array}$ & $\begin{array}{l}67 \\
(94.4)\end{array}$ & 0.202 & $\begin{array}{l}1.36 \\
(0.353- \\
5.25)\end{array}$ & 0.65 \\
\hline \multicolumn{12}{|c|}{ Critical thinking skills } \\
\hline Think critically & $\begin{array}{l}186 \\
(94.9)\end{array}$ & $62(91.2)$ & $\begin{array}{l}118 \\
(96.7)\end{array}$ & 2.69 & $\begin{array}{l}0.350 \\
(0.095- \\
1.29)\end{array}$ & 0.10 & $\begin{array}{l}114 \\
(95.8)\end{array}$ & $\begin{array}{l}66 \\
(93.0)\end{array}$ & 0.72 & $\begin{array}{l}1.73 \\
(0.482- \\
6.19)\end{array}$ & 0.40 \\
\hline
\end{tabular}

+ Significant when adjusted for clinical exposure.

*Significant when adjusted for gender. 


\begin{tabular}{|c|c|c|c|c|c|c|c|c|c|c|c|}
\hline \multirow{2}{*}{$\begin{array}{l}\text { Domains of } \\
\text { learning-"I feel } \\
\text { that TriGen has } \\
\text { helped me" }\end{array}$} & \multirow{2}{*}{$\begin{array}{l}\text { Total } \\
\text { no. } \\
\text { (\%), n } \\
=196\end{array}$} & \multirow{2}{*}{$\begin{array}{l}\text { Male } \\
\text { students, } \\
\text { no. (\%), } \\
n=68\end{array}$} & \multirow{2}{*}{$\begin{array}{l}\text { Female } \\
\text { students, } \\
\text { no.(\%), n } \\
=122\end{array}$} & \multicolumn{3}{|c|}{ Males versus females } & \multirow{2}{*}{$\begin{array}{l}\text { Year } \\
1 \text { and } \\
2, \text { no. } \\
(\%), n \\
=119\end{array}$} & \multirow{2}{*}{$\begin{array}{l}\text { Year } 3 \\
\text { and } \\
\text { above, } \\
\text { no. } \\
\text { (\%), n } \\
=71\end{array}$} & \multicolumn{3}{|c|}{ Pre-clinical versus Clinical } \\
\hline & & & & $\begin{array}{l}\text { Pearson } \\
\text { Chi- } \\
\text { Square }\end{array}$ & $\begin{array}{l}\text { Unadjusted } \\
\text { odds ratio } \\
\text { (OR) (95\% } \\
\text { confidence } \\
\text { interval) }\end{array}$ & $\begin{array}{l}P \\
\text { value }\end{array}$ & & & $\begin{array}{l}\text { Pearson } \\
\text { Chi- } \\
\text { Square }\end{array}$ & $\begin{array}{l}\text { Unadjusted } \\
\text { odds ratio } \\
\text { (OR) (95\% } \\
\text { confidence } \\
\text { interval) }\end{array}$ & $\begin{array}{l}P \\
\text { value }\end{array}$ \\
\hline $\begin{array}{l}\text { Identify social } \\
\text { issues and } \\
\text { concerns }\end{array}$ & $\begin{array}{l}192 \\
(98.0)\end{array}$ & $66(97.1)$ & $\begin{array}{l}120 \\
(98.4)\end{array}$ & 0.359 & $\begin{array}{l}0.55 \\
(0.076- \\
4.00)\end{array}$ & 0.55 & $\begin{array}{l}116 \\
(97.5)\end{array}$ & $\begin{array}{l}70 \\
(98.6)\end{array}$ & 0.267 & $\begin{array}{l}0.552 \\
(0.056- \\
5.41)\end{array}$ & 0.61 \\
\hline \multicolumn{12}{|l|}{ Action skills } \\
\hline Take action & $\begin{array}{l}186 \\
(94.9)_{2}\end{array}$ & $61(89.7)$ & $\begin{array}{l}119 \\
(97.5)\end{array}$ & 5.38 & $\begin{array}{l}0.22 \\
(0.055- \\
0.88)\end{array}$ & $0.020^{+}$ & $\begin{array}{l}113 \\
(95.0)\end{array}$ & $\begin{array}{l}67 \\
(94.4)\end{array}$ & 0.031 & $\begin{array}{l}1.12 \\
(0.306- \\
4.13)\end{array}$ & 0.86 \\
\hline $\begin{array}{l}\text { Build confidence } \\
\& \text { take on new } \\
\text { responsibilities }\end{array}$ & $\begin{array}{l}191 \\
(97.4)\end{array}$ & $64(94.1)$ & $\begin{array}{l}121 \\
(99.2)\end{array}$ & 4.37 & $\begin{array}{l}0.132 \\
(0.014- \\
1.21)\end{array}$ & 0.037 & $\begin{array}{l}117 \\
(98.3)\end{array}$ & $\begin{array}{l}68 \\
(95.8)\end{array}$ & 1.12 & $\begin{array}{l}2.58 \\
(0.421- \\
15.8)\end{array}$ & 0.29 \\
\hline \multicolumn{12}{|c|}{ Gaining of knowledge } \\
\hline $\begin{array}{l}\text { Appreciate and } \\
\text { identify gaps or } \\
\text { deficiencies in } \\
\text { the healthcare } \\
\text { system }\end{array}$ & $\begin{array}{l}185 \\
(94.4)\end{array}$ & $61(89.7)$ & $\begin{array}{l}118 \\
(96.7)\end{array}$ & 3.94 & $\begin{array}{l}0.295 \\
(0.083- \\
1.05)\end{array}$ & 0.047 & $\begin{array}{l}111 \\
(93.3)\end{array}$ & $\begin{array}{l}68 \\
(95.8)\end{array}$ & 0.508 & $\begin{array}{l}0.612 \\
(0.157- \\
2.39)\end{array}$ & 0.48 \\
\hline $\begin{array}{l}\text { Appreciate my } \\
\text { own health, } \\
\text { living condition }\end{array}$ & $\begin{array}{l}192 \\
(98.0)\end{array}$ & $66(97.1)$ & $\begin{array}{l}120 \\
(98.4)\end{array}$ & 0.359 & $\begin{array}{l}0.550 \\
(0.076- \\
4.00)\end{array}$ & 0.55 & $\begin{array}{l}117 \\
(98.3)\end{array}$ & $\begin{array}{l}69 \\
(97.2)\end{array}$ & 0.279 & $\begin{array}{l}1.70 \\
(0.234- \\
12.3)\end{array}$ & 0.60 \\
\hline $\begin{array}{l}\text { Improve my } \\
\text { general } \\
\text { knowledge } \\
\text { about healthcare }\end{array}$ & $\begin{array}{l}180 \\
(91.8)\end{array}$ & $61(89.7)$ & $\begin{array}{l}113 \\
(92.6)\end{array}$ & 0.482 & $\begin{array}{l}0.694 \\
(0.246- \\
2.00)\end{array}$ & 0.49 & $\begin{array}{l}108 \\
(90.8)\end{array}$ & $\begin{array}{l}66 \\
(93.0)\end{array}$ & 0.279 & $\begin{array}{l}0.744 \\
(0.247- \\
2.24)\end{array}$ & 0.60 \\
\hline $\begin{array}{l}\text { Enhance my } \\
\text { understanding } \\
\text { of the use of } \\
\text { public health } \\
\text { measures in } \\
\text { resource poor } \\
\text { setting }\end{array}$ & $\begin{array}{l}189 \\
(96.4)\end{array}$ & $64(94.1)$ & $\begin{array}{l}119 \\
(97.5)\end{array}$ & 1.44 & $\begin{array}{l}0.40 \\
(0.088- \\
1.86)\end{array}$ & 0.23 & $\begin{array}{l}114 \\
(95.8)\end{array}$ & $\begin{array}{l}69 \\
(97.2)\end{array}$ & 0.24 & $\begin{array}{l}0.661 \\
(0.125- \\
3.50)\end{array}$ & 0.62 \\
\hline \multicolumn{12}{|c|}{ Application of knowledge } \\
\hline $\begin{array}{l}\text { Improve my } \\
\text { clinical } \\
\text { diagnostic skills }\end{array}$ & $\begin{array}{l}160 \\
(81.6)\end{array}$ & $54(79.4)$ & $\begin{array}{l}100 \\
(82.0)\end{array}$ & 0.186 & $\begin{array}{l}0.849 \\
(0.402- \\
1.79)\end{array}$ & 0.67 & $\begin{array}{l}91 \\
(76.5)\end{array}$ & $\begin{array}{l}63 \\
(88.7)\end{array}$ & 4.35 & $\begin{array}{l}0.413 \\
(0.177- \\
0.964)\end{array}$ & $0.037^{*}$ \\
\hline $\begin{array}{l}\text { Apply what I } \\
\text { learnt in the } \\
\text { training } \\
\text { sessions (the } \\
\text { one organized } \\
\text { before the start } \\
\text { of the home } \\
\text { visits) }\end{array}$ & $\begin{array}{l}157 \\
(80.1)\end{array}$ & $57(83.8)$ & $94(77.0)$ & 1.23 & $\begin{array}{l}1.54 \\
(0.714- \\
3.34)\end{array}$ & 0.27 & $\begin{array}{l}87 \\
(73.1)\end{array}$ & $\begin{array}{l}64 \\
(90.1)\end{array}$ & 7.91 & $\begin{array}{l}0.297 \\
(0.123- \\
0.716)\end{array}$ & $0.005^{\star}$ \\
\hline + Significant when & liucter & lininal & sure. & & & & & & & & \\
\hline
\end{tabular}

When adjusted for clinical experience, female students were more likely to report gains in the area of participation in community affairs and taking action. When adjusted for gender, students in the clinical phase of their training were more likely to report gains in their clinical diagnostic skills and application of knowledge and skills learned during the training session and the ability to comprehend the moral and ethical issues in healthcare.

\section{Secondary School Students}

Most SS students felt that TriGen was beneficial across all nine FIPSE domains (Table 8). When adjusted for age, females were more likely to report gains in respect different opinion, compromise. When adjusted for gender, older students were more likely to report gains in appreciate teamwork and 
cooperation among peers, appreciate and identify gaps or deficiency in the healthcare system and enhance understanding of use of public health measures in resource poor setting. 
Table 8

FIPSE in Secondary School Students

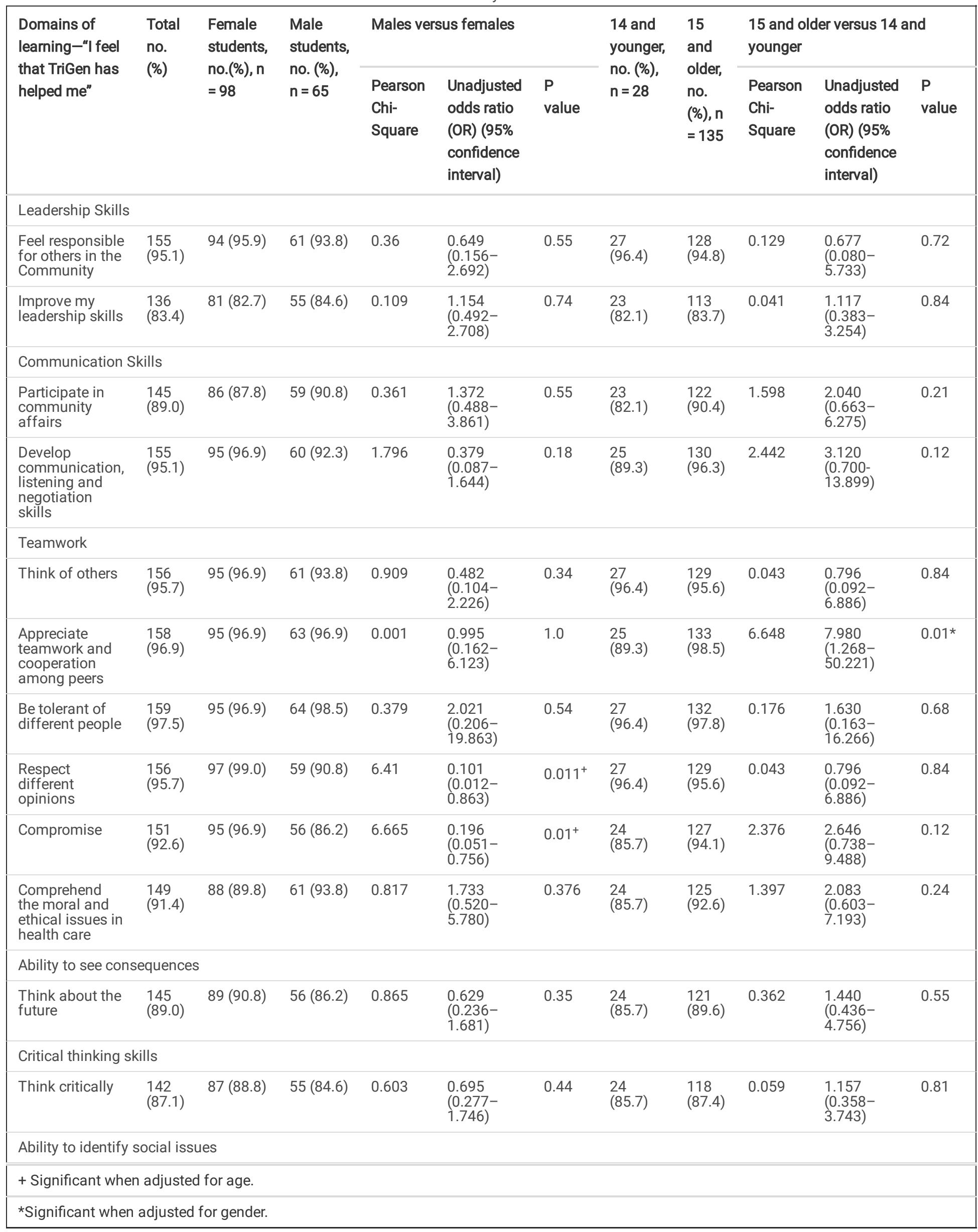




\begin{tabular}{|c|c|c|c|c|c|c|c|c|c|c|c|}
\hline \multirow{2}{*}{$\begin{array}{l}\text { Domains of } \\
\text { learning-"I feel } \\
\text { that TriGen has } \\
\text { helped me" }\end{array}$} & \multirow{2}{*}{$\begin{array}{l}\text { Total } \\
\text { no. } \\
(\%)\end{array}$} & \multirow{2}{*}{$\begin{array}{l}\text { Female } \\
\text { students, } \\
\text { no.(\%), n } \\
=98\end{array}$} & \multirow{2}{*}{$\begin{array}{l}\text { Male } \\
\text { students, } \\
\text { no. (\%), } \\
n=65\end{array}$} & \multicolumn{3}{|c|}{ Males versus females } & \multirow{2}{*}{$\begin{array}{l}14 \text { and } \\
\text { younger, } \\
\text { no. }(\%) \text {, } \\
n=28\end{array}$} & \multirow{2}{*}{$\begin{array}{l}15 \\
\text { and } \\
\text { older, } \\
\text { no. } \\
(\%), n \\
=135\end{array}$} & \multicolumn{3}{|c|}{$\begin{array}{l}15 \text { and older versus } 14 \text { and } \\
\text { younger }\end{array}$} \\
\hline & & & & $\begin{array}{l}\text { Pearson } \\
\text { Chi- } \\
\text { Square }\end{array}$ & $\begin{array}{l}\text { Unadjusted } \\
\text { odds ratio } \\
\text { (OR) (95\% } \\
\text { confidence } \\
\text { interval) }\end{array}$ & $\begin{array}{l}P \\
\text { value }\end{array}$ & & & $\begin{array}{l}\text { Pearson } \\
\text { Chi- } \\
\text { Square }\end{array}$ & $\begin{array}{l}\text { Unadjusted } \\
\text { odds ratio } \\
\text { (OR) (95\% } \\
\text { confidence } \\
\text { interval) }\end{array}$ & $\begin{array}{l}P \\
\text { value }\end{array}$ \\
\hline $\begin{array}{l}\text { Identify social } \\
\text { issues and } \\
\text { concerns }\end{array}$ & $\begin{array}{l}151 \\
(92.6)\end{array}$ & $94(95.9)$ & $57(87.7)$ & 3.878 & $\begin{array}{l}0.303 \\
(0.087- \\
1.052)\end{array}$ & 0.049 & $\begin{array}{l}24 \\
(85.7)\end{array}$ & $\begin{array}{l}127 \\
(94.1)\end{array}$ & 2.376 & $\begin{array}{l}2.646 \\
(0.738- \\
9.488)\end{array}$ & 0.12 \\
\hline \multicolumn{12}{|l|}{ Action skills } \\
\hline Take action & $\begin{array}{l}148 \\
(90.8)\end{array}$ & $90(91.8)$ & $58(89.2)$ & 0.318 & $\begin{array}{l}0.737 \\
(0.253- \\
2.140)\end{array}$ & 0.57 & $\begin{array}{l}24 \\
(85.7)\end{array}$ & $\begin{array}{l}124 \\
(91.9)\end{array}$ & 1.045 & $\begin{array}{l}1.879 \\
(0.552- \\
6.396)\end{array}$ & 0.31 \\
\hline $\begin{array}{l}\text { Build } \\
\text { confidence \& } \\
\text { take on new } \\
\text { responsibilities }\end{array}$ & $\begin{array}{l}147 \\
(90.2)\end{array}$ & $91(92.9)$ & $56(86.2)$ & 1.984 & $\begin{array}{l}0.479 \\
(0.169- \\
1.357)\end{array}$ & 0.16 & $\begin{array}{l}24 \\
(85.7)\end{array}$ & $\begin{array}{l}123 \\
(91.1)\end{array}$ & 0.763 & $\begin{array}{l}1.708 \\
(0.508- \\
5.747)\end{array}$ & 0.38 \\
\hline \multicolumn{12}{|c|}{ Gaining of knowledge } \\
\hline $\begin{array}{l}\text { Appreciate and } \\
\text { identify gaps or } \\
\text { deficiencies in } \\
\text { the healthcare } \\
\text { system }\end{array}$ & $\begin{array}{l}151 \\
(92.6)\end{array}$ & $93(94.9)$ & $58(89.2)$ & 1.84 & $\begin{array}{l}0.445 \\
(0.135- \\
1.470)\end{array}$ & 0.18 & $\begin{array}{l}22 \\
(78.6)\end{array}$ & $\begin{array}{l}129 \\
(95.6)\end{array}$ & 9.809 & $\begin{array}{l}5.864 \\
(1.734- \\
19.833)\end{array}$ & $0.002^{*}$ \\
\hline $\begin{array}{l}\text { Appreciate my } \\
\text { own health, } \\
\text { living condition }\end{array}$ & $\begin{array}{l}158 \\
(96.9)\end{array}$ & 97 (99.0) & $61(93.8)$ & 3.464 & $\begin{array}{l}0.157 \\
(0.017- \\
1.440)\end{array}$ & 0.063 & $\begin{array}{l}27 \\
(96.4)\end{array}$ & $\begin{array}{l}131 \\
(97.0)\end{array}$ & 0.029 & $\begin{array}{l}1.213 \\
(0.130- \\
11.282)\end{array}$ & 0.87 \\
\hline $\begin{array}{l}\text { Improve my } \\
\text { general } \\
\text { knowledge } \\
\text { about } \\
\text { healthcare }\end{array}$ & $\begin{array}{l}153 \\
(93.9)\end{array}$ & $92(93.9)$ & $61(93.8)$ & 0.001 & $\begin{array}{l}0.995 \\
(0.269- \\
3.671)\end{array}$ & 0.99 & $\begin{array}{l}25 \\
(89.3)\end{array}$ & $\begin{array}{l}128 \\
(94.8)\end{array}$ & 1.231 & $\begin{array}{l}2.194 \\
(0.531- \\
9.067)\end{array}$ & 0.27 \\
\hline $\begin{array}{l}\text { Enhance my } \\
\text { understanding } \\
\text { of the use of } \\
\text { public health } \\
\text { measures in } \\
\text { resource poor } \\
\text { setting }\end{array}$ & $\begin{array}{l}147 \\
(90.2)\end{array}$ & $92(93.9)$ & $55(84.6)$ & 3.787 & $\begin{array}{l}0.359 \\
(0.124- \\
1.041)\end{array}$ & 0.052 & $\begin{array}{l}22 \\
(78.6)\end{array}$ & $\begin{array}{l}125 \\
(92.6)\end{array}$ & 5.15 & $\begin{array}{l}3.409 \\
(1.125- \\
10.333)\end{array}$ & $0.023^{*}$ \\
\hline \multicolumn{12}{|c|}{ Application of knowledge } \\
\hline $\begin{array}{l}\text { Improve my } \\
\text { caregiving skills } \\
\text { (e.g. blood } \\
\text { pressure } \\
\text { measurement, } \\
\text { capillary blood } \\
\text { glucose } \\
\text { measurement) }\end{array}$ & $\begin{array}{l}149 \\
(91.4)\end{array}$ & $90(91.8)$ & $59(90.8)$ & 0.057 & $\begin{array}{l}0.874 \\
(0.289- \\
2.648)\end{array}$ & 0.81 & $\begin{array}{l}24 \\
(85.7)\end{array}$ & $\begin{array}{l}125 \\
(92.6)\end{array}$ & 1.397 & $\begin{array}{l}2.083 \\
(0.603- \\
7.193)\end{array}$ & 0.24 \\
\hline $\begin{array}{l}\text { Apply what I } \\
\text { learnt in the } \\
\text { training } \\
\text { sessions (the } \\
\text { one organized } \\
\text { before the start } \\
\text { of the home } \\
\text { visits) }\end{array}$ & $\begin{array}{l}138 \\
(84.7)\end{array}$ & $80(81.6)$ & $58(89.2)$ & 1.737 & $\begin{array}{l}1.864 \\
(0.731- \\
4.754)\end{array}$ & 0.19 & $\begin{array}{l}22 \\
(78.6)\end{array}$ & $\begin{array}{l}116 \\
(85.9)\end{array}$ & 0.966 & $\begin{array}{l}1.665 \\
(0.598- \\
4.640)\end{array}$ & 0.33 \\
\hline + Significant whe & diuct & age. & & & & & & & & & \\
\hline
\end{tabular}

Program feedback 


\section{Healthcare undergraduates}

Majority of the healthcare undergraduates felt more prepared for their practice as healthcare professionals in the future. $92.4 \%$ are now more aware of the problems faced by the older persons. $91.9 \%$ would recommend the program to their friends. (Table 9)

Table 9

Quantitative Feedback

\begin{tabular}{|c|c|c|}
\hline & $\begin{array}{l}\% \text { of healthcare undergraduates who agreed } \\
(n=185 \text { unless otherwise stated) }(95 \% \mathrm{Cl})\end{array}$ & $\begin{array}{l}\% \text { of students who } \\
\text { agreed }(n=172)(95 \% \\
\text { Cl) }\end{array}$ \\
\hline The multi-disciplinary meetings were useful for learning & $72.8(65.7-79.9)(n=169)$ & \\
\hline $\begin{array}{l}\text { I am more prepared for my practice as a healthcare professional in the } \\
\text { future. }\end{array}$ & $80.5(74.6-85.9)$ & \\
\hline $\begin{array}{l}\text { I am inspired and empowered to start something new to fulfill a social } \\
\text { need }\end{array}$ & $75.1(68.1-80.5)$ & \\
\hline I have achieved my personal goals set at the start of the cycle & $76.2(69.7-82.2)$ & \\
\hline $\begin{array}{l}\text { I better appreciate the importance of inter-professional collaboration in } \\
\text { the care of patients }\end{array}$ & $91.6(86.3-96.8)(n=95)$ & \\
\hline I am now more confident with communicating with the elderly. & & $62.2(55.2-69.8)$ \\
\hline $\begin{array}{l}\text { The lessons learnt during the home visits are applicable to me and my } \\
\text { family }\end{array}$ & & $75.0(68.0-81.4)$ \\
\hline $\begin{array}{l}\text { I will bring back the lessons learnt and educate my family members } \\
\text { regarding the importance of healthy lifestyle and health screening }\end{array}$ & & $64.0(56.4-71.5)$ \\
\hline The curriculum is useful for my learning & & $64.0(57.0-71.5)$ \\
\hline I am now more confident in providing basic caregiving skills & $90.3(85.9-94.1)$ & $65.7(58.7-72.7)$ \\
\hline I am now more aware of the problems faced by the elderly & $92.4(88.6-96.2)$ & $83.1(77.9-88.4)$ \\
\hline I would recommend TriGen to my friends & $91.9(87.6-95.7)$ & $91.3(86.6-95.3)$ \\
\hline
\end{tabular}

\section{Secondary School Students}

$83.1 \%$ of SS students are more aware of problems faced by the older persons and $91.3 \%$ would recommend the program to their friends. (Table 9 )

\section{Patients' outcomes}

Table 10 describes the demographic of our patients. There were 116 patients who participated in the program. The mean age is 73.5 years-old. The mean age-adjusted Charlson co-morbidity index is 9.1 . 
Table 10

Patient Demographics

\begin{tabular}{|c|c|c|}
\hline Demographic & Variables & Numbers (Percentages) \\
\hline Age & Mean 73.5 (54-95 years) & \\
\hline \multirow[t]{2}{*}{ Gender } & Males & $58(54.7)$ \\
\hline & Female & $48(45.3)$ \\
\hline \multirow[t]{4}{*}{ Race } & Chinese & $71(67.0)$ \\
\hline & Malay & $6(5.7)$ \\
\hline & Indian & $26(24.5)$ \\
\hline & Others & $3(2.8)$ \\
\hline Charlson Comorbidities Index & Mean 9.1 (SD 2.9) & \\
\hline Lawton IADL & Mean 4.4 (SD 2.4) & \\
\hline \multirow[t]{2}{*}{ Alone } & Yes & $12(19.0)$ \\
\hline & No & $51(81.0)$ \\
\hline \multirow[t]{6}{*}{ Housing } & 1-room & $13(21.0)$ \\
\hline & 2-room & $4(6.5)$ \\
\hline & 3-room & $22(35.5)$ \\
\hline & 4-room & $18(29.0)$ \\
\hline & 5-room & $4(6.5)$ \\
\hline & Others & $1(1.6)$ \\
\hline \multirow[t]{2}{*}{ Financial } & Yes & $40(39.2)$ \\
\hline & No & $62(60.8)$ \\
\hline \multirow[t]{5}{*}{ Carer } & Self & $27(42.9)$ \\
\hline & Spouse & $8(12.7)$ \\
\hline & Children & $26(41.3)$ \\
\hline & Grandchildren & $1(1.6)$ \\
\hline & Siblings & $1(1.6)$ \\
\hline
\end{tabular}

A Wilcoxon signed-rank test showed a statistically significant decrease in hospital admission rates during the 6 months period before the program and the 6 months period after from a median of 1 visit to 0 visit. $(Z=2.72, p=0.006)$. A Wilcoxon signed-rank test showed a statistically significant decrease in emergency department visits during the 6 months period before the program and 6 months period after from a median of 1 visit to 1 visit. $(Z=2.91, p=0.004)$

A total of 51 patients answered the patient feedback survey. The majority (> 80\%) felt less lonely and happier because of the home visits. Most (> $50 \%)$ felt that they have changed their lifestyle for the better and feel more confident taking care of their own health as a result of the home visit. (Table 11) 
Table 11

Patient Feedback

\begin{tabular}{|c|c|c|c|c|}
\hline Because of the home visits, I .. & $\begin{array}{l}\text { Agree (\% of } \\
\text { respondents) }\end{array}$ & Neutral & Disagree & $\begin{array}{l}\text { Did not answer (\% of } \\
\text { all patients) }\end{array}$ \\
\hline $\begin{array}{l}\text { I understand more about my health problems because of the } \\
\text { home visits }\end{array}$ & $35(71.4)$ & $8(16.3)$ & $6(12.2)$ & $57(53.8)$ \\
\hline $\begin{array}{l}\text { I feel more confident in taking care of my own health } \\
\text { because of the home visits }\end{array}$ & $34(68.0)$ & $12(24.0)$ & $4(8.0)$ & $56(52.8)$ \\
\hline $\begin{array}{l}\text { I have changed my lifestyle (e.g. diet, exercise, leaving the } \\
\text { house more often, etc.) }\end{array}$ & $25(50.0)$ & $13(26.0)$ & $12(24.0)$ & $56(52.8)$ \\
\hline $\begin{array}{l}\text { I want to continue to improve my health because of the } \\
\text { home visits }\end{array}$ & $30(61.2)$ & $11(22.4)$ & $8(16.3)$ & $57(53.8)$ \\
\hline $\begin{array}{l}\text { I feel less afraid to ask questions about my health because } \\
\text { of the home visits }\end{array}$ & $26(53.1)$ & $12(24.5)$ & $11(22.4)$ & $57(53.8)$ \\
\hline I enjoyed the activities done during the home visits & $48(96.0)$ & $2(4.0)$ & $0(0.0)$ & $56(52.8)$ \\
\hline I feel less lonely because of the home visits & $42(84.0)$ & $6(12.0)$ & $2(4.0)$ & $56(52.8)$ \\
\hline I feel happier because of the home visits & $48(94.1)$ & $3(5.9)$ & & $55(51.9)$ \\
\hline I look forward to the home visits & $44(86.3)$ & $6(11.8)$ & $1(2.0)$ & $55(51.9)$ \\
\hline $\begin{array}{l}\text { I would like to continue the home visits with a different group } \\
\text { of students }\end{array}$ & $32(65.3)$ & $10(20.4)$ & $7(14.3)$ & $57(53.8)$ \\
\hline The students were respectful & $49(96.1)$ & $2(3.9)$ & $0(0.0)$ & $55(51.9)$ \\
\hline I found it easy to talk to the students & $42(82.4)$ & $8(15.7)$ & $1(2.0)$ & $55(51.9)$ \\
\hline I made friends with the students & $37(72.5)$ & $12(23.5)$ & $2(3.9)$ & $55(51.9)$ \\
\hline My family/caregivers enjoy the home visits & $26(70.3)$ & $10(27.0)$ & $1(2.7)$ & $69(65.1)$ \\
\hline $\begin{array}{l}\text { My family/caregivers are engaged by the team leaders and } \\
\text { students during the home visits }\end{array}$ & $23(69.7)$ & $9(27.3)$ & $1(3.0)$ & $73(68.9)$ \\
\hline \multirow[t]{2}{*}{ It was easy to schedule each home visit } & $40(78.4)$ & $8(15.7)$ & $3(5.9)$ & 55 (51.9) \\
\hline & Just nice & $\begin{array}{l}\text { Too short / Too } \\
\text { infrequent }\end{array}$ & $\begin{array}{l}\text { Too long / Too } \\
\text { frequent }\end{array}$ & Missing \\
\hline The duration of home visits is & $45(88.2)$ & $5(9.8)$ & $1(2.0)$ & $55(51.9)$ \\
\hline The frequent of the home visits is & $43(86.0)$ & $7(14.0)$ & $0(0.0)$ & $56(52.8)$ \\
\hline The number of students per visit is & $51(100.0)$ & $0(0.0)$ & $0(0.0)$ & $55(51.9)$ \\
\hline
\end{tabular}

\section{Discussion}

\section{Reducing Ageism}

To our knowledge, this is the first paper that discusses a longitudinal student-initiated, inter-generational, inter-professional home visit program which enables healthcare undergraduates and lay students (SS students) to provide holistic care to the older persons in the community through a service-learning approach.

TriGen decreased ageist attitudes amongst both healthcare undergraduates and SS students, as evidenced by significant increases in KOP scores for both groups. This effect is greater amongst the healthcare undergraduates, possibly because of their greater involvement in the care of the older persons compared to the SS students. This increase in KOP scores were found in all subgroups of participants (Table 3 and 4 ).

TriGen also increased knowledge about the older persons amongst SS students (mean increase of $0.8 \pm 0.5$, $p$-value $=0.005$ ), but not healthcare undergraduates.

Levy et al. proposed a theoretical model to reduce ageism which consisted of education and positive contact with the older persons (34). We propose that our program supports the validity of this theoretical model. 
Firstly, our program employed education on aging to dispel negative and inaccurate images of older adulthood. There was a correlation between change in KOP scores and Palmore's FAQ scores amongst the SS students suggesting that an increase in knowledge of the older persons may reduce ageism. While there was no statistical increase in the Palmore's FAQ score for healthcare undergraduates, there was a significant correlation between change in KOP score and change in Palmore's score. $(r=0.266, p<0.001)$. Our qualitative data showed that both healthcare undergraduates and SS students demonstrated greater understanding of the older person. These qualitative data will be published in a separate paper. This is supported by studies that demonstrated a more accurate knowledge of aging is associated with less ageist attitudes (35). Moreover, educational efforts to increase knowledge of the aging do result in a reduction in ageist attitudes $(36,37)$.

Secondly, TriGen provided positive contact with the older persons. This is based on the intergroup contact hypothesis which proposes that negative intergroup attitudes stem in part from lack of positive contact between group members $(38,39)$. In the qualitative analysis, the healthcare undergraduates and SS students reported that they had a very meaningful experience with the older persons and many enjoyed the intergenerational bonding. Amongst SS students, those who spent more time interacting with the elderly in TriGen had a greater decrease in ageist attitudes, supporting the contact theory.

Lastly, we propose TriGen reduced ageism because it developed empathy and understanding of person-centric care amongst our participants. Most of our participants (more than $90 \%$ ) reported gain in the skill of being able to think of others and being tolerant of different people (Table 7 and 8 ). Moreover, in our qualitative data amongst the healthcare undergraduates, a major theme is that healthcare undergraduates learned to see the patient beyond their diseases and conditions to see them as individuals with unique life stories; they also reported developing empathy. Amongst SS students, a major theme is the development of empathy.

The healthcare undergraduates have a higher pre-intervention mean KOP score (133.0 \pm 2.9$)$ compared to the SS students (127.4 \pm 1.8$)$, perhaps reflecting the tendency for less ageist individuals to join the healthcare professions and the greater amount of geriatric education and experiences working with older persons the healthcare undergraduates get. The healthcare undergraduates in this study had similar positive attitudes towards the older person as compared to Year 1 and Year 3 medical students in a study by Cheong et al (mean KOP score was $135.2 \pm 14.9$ and $138.2 \pm 13.5$ for the Year 1 and Year 3 medical students respectively) (40).

When compared with the junior doctors comprising House Officers, Medical Officers and Registrar in a tertiary hospital described in a previous study done by Lui et al., our healthcare undergraduates had a much higher pre-intervention KOP score (133.0 \pm 2.9$)$ (41). The mean KOP score of the doctors in that study is $114.4 \pm 9.0$. Empathy level are known to decrease as training progresses amongst medical students and residents (23-26, 42). There is possibly such a similar trend in ageism (22). However, current data conflicts with one study demonstrating a possible increase in ageism as training progresses amongst junior doctors, and another study reporting a possible decrease in ageism with increasing years of seniority amongst medical students. In both studies, the trend was not statistically significant $(40,41)$.

We would expect that those who live with their grandparents and/or who had previous experiences volunteering with the older persons would have higher pre-intervention mean KOP scores. Indeed, healthcare undergraduates who live with their grandparents had higher mean pre-intervention KOP scores. However, this is not seen amongst SS students. Interestingly, previous experiences volunteering with older persons were not associated with significant differences in mean pre-intervention KOP scores amongst both healthcare undergraduates and SS students; reasons why deserve to be explored in future studies.

\section{Personal Development and Gaining of Skills}

Participants found that TriGen was effective in increasing their understanding of all 9 domains of the FIPSE survey instruments. This is likely because TriGen is a student-initiated project with high levels of student involvement in all phases of the project from conceptualization, and planning to implementation. Moreover, the longitudinal nature of TriGen conducted over 6 months provided for ample opportunities to develop skills and inculcate values. There were some differences in the perceived educational value for students of differing gender, age and clinical experience, but it is known that these can affect students' attitudes towards learning (43-45). In contrast to reports by various authors in the Asian and Western setting that healthcare students with no prior clinical exposure are more likely to have higher perceived educational value (46-48), we found that the healthcare undergraduates with clinical experience gained more in being able to apply what they have learned in the training sessions to the home visits and improved clinical diagnostic skills. We postulate that this is because TriGen is a longitudinal program involving patients with complex care needs who have frequent admissions. Hence, healthcare undergraduates with more clinical experience were more likely to be able to grasp and process the complexities.

Healthcare undergraduates were more likely to perceive TriGen to have helped them improve their leadership skills. Approximately $10 \%$ more healthcare undergraduates as compared to SS students agreed to the statement "TriGen has helped me to improve my leadership skills." This is likely due to the nature of the involvement of both groups in TriGen: the healthcare undergraduates, as the leaders of each visitation team, were directly accountable to the community nurses and other hospital healthcare professionals in KTPH, and took charge of representing the patient's interests in multi-disciplinary meetings. The SS students' role were relatively more passive, and they mostly led by the healthcare undergraduates in 
interacting with the older persons in home visits. As such, they had a much smaller degree of ownership and responsibility in the care of the older persons.

\section{Impact on the patients}

Patients were observed to have a reduction in the number of hospital admissions and emergency department visits after participating in TriGen. The evidence suggests that integrated geriatrics care provided through home visits may reduce acute hospital use (4-6). Our study suggests that a student-initiated, intergenerational, interprofessional program supervised by healthcare professionals can potentially achieve similar efficacy as a home visit program run by a professional geriatrics unit. Most patients reported that they have made positive changes to their lifestyles and are more confident of self-care as a result of the program. Also, another possible explanation for the reduction in utilization of healthcare resources could be secondary to the improved psychological and social wellbeing (49). Many reported that they enjoyed the home visits and felt less lonely and happier as a result of the program.

\section{Limitations}

Our study has several limitations. First, as participation for both undergraduates and SS students was voluntary, the participants were likely to be self-selected and this would reduce the generalizability of our findings. However, it is noted that the mean pre-intervention KOP score is similar to that reported in other cohorts of medical students and nursing students. Second, the learning outcomes were self-reported. Third, we have no longitudinal data on the long-term learning outcomes. Lastly, the healthcare resources utilization data are not controlled and the reduction in utilization of healthcare resources cannot be conclusively attributed to the program.

\section{Conclusion}

In summary, TriGen demonstrates the potential of a student-initiated, longitudinal, inter-generational and inter-professional home visit program to reduce ageism, develop soft skills, inculcate values amongst lay volunteers (SS students) and healthcare undergraduates. In addition, TriGen potentially reduces hospital admissions and emergency department visits, and loneliness amongst frequently admitted older patients. We hope that our experience will encourage other institutions and communities to adopt this concept that we have shown to be feasible and impactful.

\section{Declarations}

\section{Ethical approval:}

The institutional review board of National University of Singapore and the domain specific review board of Khoo Teck Puat Hospital granted ethical approval for this study.

\section{Consent for publication:}

Not applicable.

\section{Availability of data and materials:}

The datasets used and/or analysed during the current study are available from the corresponding author on reasonable request.

Competing interests: None.

\section{Funding/Support:}

National University of Singapore, Yong Loo Lin School of Medicine, Dean's Office; the North West Community Development Council; Khoo Teck Puat Hospital, Singapore provided funding support for the purchase of medical consumables, refreshments and logistics for the program.

\section{Acknowledgements:}

The authors would like to thank the Tri-Generational HomeCare Organizing Committee from 2014 to 2018 for supporting the study. They would like to extend their thanks to the National University of Singapore, Yong Loo Lin School of Medicine, Dean's Office; the North West Community Development Council; Khoo Teck Puat Hospital, Singapore; Geriatric Education and Research Institute, Singapore. Finally, they would like to thank the volunteers for their generosity and the patients for their hospitality.

\section{Authors Contributions:}


KYYN, GL, AJYT, JQC, SML, CHW, GCHK conceived and designed the study. KYYN, GL, AJYT, JQC, SML, AS, KSY, NHWN collected data and analysed the data. All authors interpreted the data and were involved in the development, review and approval of the manuscript.

\section{Authors' Information:}

Dr Kennedy NG is Senior Resident, Department of Medical Oncology, National Cancer Centre Singapore, Singapore

Dr Gloria LEUNG is House Officer, Department of Internal Medicine, Changi General Hospital, Singapore

Dr Angeline Jie-Yin TEY is Medical Officer, Department of Intensive Care Medicine, Sengkang General Hospital, Singapore

Dr Jia Quan CHAUNG is Medical Officer, Changi Naval Base, Singapore Armed Forces, Singapore

Dr Si Min LEE is Internal Medicine Resident, Department of General Medicine, Tan Tock Seng Hospital, Singapore

Dr Amrish SOUNDARARAJAN is Medical Officer, Tekong Island Medical Centre, Singapore Armed Forces, Singapore

Dr Ka Shing YOW is House Officer, Department of General Surgery, Changi General Hospital

Dr Nerice Heng Wen NGIAM is Medical Officer, Department of Internal Medicine, Singapore General Hospital

Dr Tang Ching LAU is Associate Professor, Vice-Dean (Education), Department of Medicine, Yong Loo Lin School of Medicine, National University of Singapore, Vice-Chairman, Medical Board, National University Hospital, Singapore

Dr Sweet Fun WONG is Chief Transformation Officer, Deputy Chairman, Medical Board, Clinical Director, Populational Health and Community Transformation, Khoo Teck Puat Hospital

Dr Chek Hooi WONG is Adjunct Assistant Professor, Health Services and Systems Research, Duke-NUS Medical School, Singapore; Senior Consultant, Department of Geriatric Medicine, Khoo Teck Puat Hospital, Singapore; Deputy Executive Director, Geriatric Education and Research Institute, Singapore

A/Prof Gerald C H KOH is Associate Professor, Saw Swee Hock School of Public Health, NUS, NUHS.

\section{References}

1. Older Singaporeans to double by 2030: National Population and Talent Division, Strategy Group, Prime Minister's Office; 2016 [Available from: https://www.population.sg/articles/older-singaporeans-to-double-by-2030.

2. Healthcare Statistics: Hospital Admission and Outpatient Attendance. In: Ministry of Health S, editor. Singapore2019.

3. Nagaya Y, Dawson A. Community-based care of the elderly in rural Japan: a review of nurse-led interventions and experiences. Journal of community health. 2014;39(5):1020-8.

4. Elkan R, Egger M, Kendrick D, Dewey M, Hewitt M, Robinson J, et al. Effectiveness of home based support for older people: systematic review and meta-analysis. BMJ. 2001;323(7315):719.

5. Di Pollina L, Guessous I, Petoud V, Combescure C, Buchs B, Schaller P, et al. Integrated care at home reduces unnecessary hospitalizations of community-dwelling frail older adults: a prospective controlled trial. 2017;17(1):53.

6. Rosenberg T. Acute Hospital Use, Nursing Home Placement, and Mortality in a Frail Community-Dwelling Cohort Managed with Primary Integrated Interdisciplinary Elder Care at Home. 2012;60(7):1340-6.

7. Marutani M, Tamura S, Miyazaki M, Amamiya Y. Evaluation of culturally appropriate health counselling to prevent lifestyle-related diseases and its modification for practical use as the new ABC model of culturally appropriate counselling for Japanese public health nurses. International journal of nursing practice. 2013;19 Suppl 2:39-49.

8. Oyama H, Goto M, Fujita M, Shibuya H, Sakashita T. Preventing elderly suicide through primary care by community-based screening for depression in rural Japan. Crisis. 2006;27(2):58-65.

9. Oyama H, Ono Y, Watanabe N, Tanaka E, Kudoh S, Sakashita T, et al. Local community intervention through depression screening and group activity for elderly suicide prevention. Psychiatry and clinical neurosciences. 2006;60(1):110-4.

10. Lofvenmark C, Mattiasson AC, Billing E, Edner M. Perceived loneliness and social support in patients with chronic heart failure. European journal of cardiovascular nursing : journal of the Working Group on Cardiovascular Nursing of the European Society of Cardiology. 2009;8(4):251-8.

11. Petitte T, Mallow J, Barnes E, Petrone A, Barr T, Theeke L. A Systematic Review of Loneliness and Common Chronic Physical Conditions in Adults. The open psychology journal. 2015;8(Suppl 2):113-32.

12. Cherubini A, Corsonello A, Lattanzio F. Underprescription of beneficial medicines in older people: causes, consequences and prevention. Drugs \& aging. 2012;29(6):463-75.

Page $25 / 27$ 
13. Schroyen S, Adam S, Jerusalem G, Missotten P. Ageism and its clinical impact in oncogeriatry: state of knowledge and therapeutic leads. Clinical interventions in aging. 2014;10:117-25.

14. Markopoulos C, van de Water W. Older patients with breast cancer: is there bias in the treatment they receive? Therapeutic advances in medical oncology. 2012;4(6):321-7.

15. Ryan EB, Butler RN. Communication, Aging, and Health: Toward Understanding Health Provider Relationships with Older Clients. Health Communication. 1996;8(3):191-7.

16. Jackson SE, Hackett RA, Steptoe A. Associations between age discrimination and health and wellbeing: cross-sectional and prospective analysis of the English Longitudinal Study of Ageing. The Lancet Public Health. 2019;4(4):e200-e8.

17. Yu CY, Chen KM. Experiencing simulated aging improves knowledge of and attitudes toward aging. Journal of the American Geriatrics Society. 2012;60(5):957-61.

18. Boswell SS. "Old People are Cranky": Helping Professional Trainees' Knowledge, Attitudes, Aging Anxiety, and Interest in Working with Older Adults. Educational Gerontology. 2012;38(7):465-72.

19. Lytle A, Levy SR. Reducing Ageism: Education About Aging and Extended Contact With Older Adults. Gerontologist. 2017.

20. Koh LC. Student attitudes and educational support in caring for older people--a review of literature. Nurse education in practice. 2012;12(1):1620.

21. Ryan A, Melby V, Mitchell L. An evaluation of the effectiveness of an educational and experiential intervention on nursing students' attitudes towards older people. International journal of older people nursing. 2007;2(2):93-101.

22. Slevin OD. Ageist attitudes among young adults: implications for a caring profession. Journal of advanced nursing. 1991;16(10):1197-205.

23. Bellini LM, Baime M, Shea JA. Variation of mood and empathy during internship. JAMA. 2002;287(23):3143-6.

24. Hojat M, Vergare MJ, Maxwell K, Brainard G, Herrine SK, Isenberg GA, et al. The Devil is in the Third Year: A Longitudinal Study of Erosion of Empathy in Medical School. 2009;84(9):1182-91.

25. Newton BW, Barber L, Clardy J, Cleveland E, O'Sullivan P. Is There Hardening of the Heart During Medical School? 2008;83(3):244-9.

26. West CP, Huntington JL, Huschka MM, Novotny PJ, Sloan JA, Kolars JC, et al. A Prospective Study of the Relationship between Medical Knowledge and Professionalism among Internal Medicine Residents. 2007;82(6):587-92.

27. Bertakis KD, Azari R. Patient-centered care is associated with decreased health care utilization. Journal of the American Board of Family Medicine : JABFM. 2011;24(3):229-39.

28. Jo Delaney L. Patient-centred care as an approach to improving health care in Australia. Collegian. 2018;25(1):119-23.

29. Jacoby B. Building Partnerships for Service Learning. 1 ed: Jossey-Bass; Jun 2003.

30. Jacoby B. Service-learning in higher eudcation: concepts and practices. 1 ed. San Francisco: Jossey-Bass; 1996.

31. Kogan N. Attitudes toward old people: the development of a scale and an examination of correlates. Journal of abnormal and social psychology. 1961;62:44-54.

32. Palmore E. Facts on aging. A short quiz. Gerontologist. 1977;17(4):315-20.

33. Janet Eyler DEGJ, Alexander W. Astin. Where's the Learning in Service-Learning?: Jossey-Bass; 1999.

34. Levy SR. Toward Reducing Ageism: PEACE (Positive Education about Aging and Contact Experiences) Model. The Gerontologist. 2018;58(2):226-32.

35. Wurtele SK, Maruyama L. Changing Students' Stereotypes of Older Adults. 2013;40(1):59-61.

36. Ragan AM, Bowen AM. Improving Attitudes Regarding the Elderly PopulationThe Effects of Information and Reinforcement for Change. The Gerontologist. 2001;41(4):511-5.

37. Gerald Ch Koh RAM, Wee Shiong Lim, Zubair Amin. The Knowledge-Attitude Dissociation in Geriatric Education: Can It be Overcome? . Ann Acad Med Singapore. 2012;41:383 - 9.

38. Pettigrew TF, Tropp, L.R. A meta-analytic test of intergroup contact theory. Journal of Personality and Social Psychology. 2006;90:751-83.

39. Allport GW. The nature of prejudice. Cambridge, Massachusetts Addison-Wesley Publishing Company; 1954.

40. Seng Kwing Cheong TYW, Gerald CH Koh. Attitudes Towards the Elderly among Singapore Medical Students. Ann Acad Med Singapore. 2009;38:857-61.

41. Nai Lee Lui CHW. Junior Doctors' Attitudes Towards Older Adults and its Correlates in a Tertiary-care Public Hospital. Ann Acad Med Singapore. 2009;38:125 - 9.

42. Bellini LM, Shea JA. Mood Change and Empathy Decline Persist during Three Years of Internal Medicine Training. 2005;80(2):164-7.

43. Crandall SJ, Davis SW, Broeseker AE, Hildebrandt C. A longitudinal comparison of pharmacy and medical students' attitudes toward the medically underserved. American journal of pharmaceutical education. 2008;72(6):148-

44. Lee KH, Seow A, Luo N, Koh D. Attitudes towards the doctor-patient relationship: a prospective study in an Asian medical school. 2008;42(11):1092-9.

Page 26/ 27 
45. Hoppe A PE, Birgegård G. Medical interns' view of their undergraduate medical education in Uppsala: an alumnus study with clear attitude differences between women and men. Med Teach. 2009;31:426-32.

46. Liang En W, Koh GC-H, Lim VKG. Caring for Underserved Patients Through Neighborhood Health Screening: Outcomes of a Longitudinal, Interprofessional, Student-Run Home Visit Program in Singapore. 2011;86(7):829-39.

47. Clark DL MA, Wallace D, Pierrel S, Buck DS. A multidisciplinary, learner-centered, student-run clinic for the homeless. Fam Med. 2003;35:394-7.

48. O'Toole TP, Hanusa BH, Gibbon JL, Boyles SH. Experiences and attitudes of residents and students influence voluntary service with homeless populations. Journal of general internal medicine. 1999;14(4):211-6.

49. Longman J, Passey M, Singer J, Morgan G. The role of social isolation in frequent and/or avoidable hospitalisation: rural community-based service providers' perspectives. Australian Health Review. 2013;37(2):223-31. 